\title{
Weak, Strong and Linear Convergence of the CQ-Method Via the Regularity of Landweber Operators
}

\author{
Andrzej Cegielski, Simeon Reich and Rafał Zalas
}

December 19, 2018

\begin{abstract}
We consider the split convex feasibility problem in a fixed point setting. Motivated by the well-known CQ-method of Byrne (2002), we define an abstract Landweber transform which applies to more general operators than the metric projection. We call the result of this transform a Landweber operator. It turns out that the Landweber transform preserves many interesting properties. For example, the Landweber transform of a (quasi/firmly) nonexpansive mapping is again (quasi/firmly) nonexpansive. Moreover, the Landweber transform of a (weakly/linearly) regular mapping is again (weakly/linearly) regular. The preservation of regularity is important because it leads to (weak/linear) convergence of many CQ-type methods.
\end{abstract}

Keywords: CQ-method, linear rate, split feasibility problem.

AMS Subject Classification: 47J25, 47N10, 49N45

\section{Introduction}

Let $\mathcal{H}_{1}$ and $\mathcal{H}_{2}$ be two real Hilbert spaces and let $A: \mathcal{H}_{1} \rightarrow \mathcal{H}_{2}$ be a nonzero bounded linear operator.

The split convex feasibility problem (SCFP) is to

$$
\text { find } x \in C \quad \text { such that } \quad A x \in Q \text {, }
$$

where $C \subseteq \mathcal{H}_{1}$ and $Q \subseteq \mathcal{H}_{2}$ are nonempty, closed and convex. In this paper we assume that the SCFP has at least one solution, that is, $C \cap A^{-1}(Q) \neq \emptyset$, and that

$$
C:=\operatorname{Fix} S \text { and } Q:=\operatorname{Fix} T,
$$

for some given operators $S$ and $T$. The SCFP was introduced by Censor and Elfving [CE94] for $\mathcal{H}_{1}=\mathbb{R}^{m}$ and $\mathcal{H}_{2}=\mathbb{R}^{n}$ and has attracted a lot of attention since then. Before describing the contribution of our paper, we briefly recall a few results and methods which have had significant impact on this field.

\section{Related work}

Among various methods designed for solving (1.1), the most celebrated one is the $C Q$-method of Byrne [Byr02] defined by

$$
x_{0} \in \mathcal{H}_{1} ; \quad x_{k+1}:=P_{C}\left(x_{k}+\frac{\lambda_{k}}{\|A\|^{2}} A^{*}\left(P_{Q}\left(A x_{k}\right)-A x_{k}\right)\right), \quad k=0,1,2, \ldots,
$$

where $\lambda_{k} \in[\varepsilon, 2-\varepsilon]$ for some $\varepsilon \in(0,1), P_{C}$ and $P_{Q}$ are the metric projections onto $C$ and $Q$, respectively, and $A^{*}: \mathcal{H}_{2} \rightarrow \mathcal{H}_{1}$ is the adjoint operator to $A$. The above method was shown to converge to a minimizer of

$$
f(x):=\frac{1}{2}\left\|P_{Q}(A x)-A x\right\|^{2}
$$

over $C$, assuming that such a minimizer exists. In the consistent case the limit point becomes a member of $C \cap A^{-1}(Q)$. As it has already been mentioned by Byrne, a special case of the method (1.3), with 
$C=\mathbb{R}^{m}$ and $Q=\{b\} \subset \mathbb{R}^{n}$, was introduced by Landweber in [Lan51]. Therefore, the CQ-method is sometimes referred to as a projected Landweber method; see, for example, [PB97, JEKC06, ZC10] and [Ceg12, Chapter 5].

Because of the differentiability of the squared distance function, the $C Q$-method can be viewed as the projected gradient method (PGM)

$$
x_{0} \in \mathcal{H}_{1} ; \quad x_{k+1}:=P_{C}\left(x_{k}-\frac{\lambda_{k}}{L} \nabla f\left(x_{k}\right)\right), \quad k=0,1,2, \ldots,
$$

with a convex and differentiable $f$, where $L=\|A\|^{2}$ is a Lipschitz constant of $\nabla f$. The convergence analysis of the PGM can be found, for example, in [Pol87, Chapter VII] with the constant parameter $\lambda_{k}=\lambda \in(0,2)$. Depending on the choice of the objective $f$ in (1.5), one can consider various extensions of the basic CQ-method. For example, in the case of the multiple-set split convex feasibility problem (MSSCFP) [CEKB05], which is to

$$
\text { find } x \in C \cap \bigcap_{i=1}^{m} C_{i} \quad \text { such that } A x \in \bigcap_{j=1}^{n} Q_{j},
$$

the following objective

$$
f(x):=\frac{1}{2} \sum_{i=1}^{m} \alpha_{i}\left\|P_{C_{i}} x-x\right\|^{2}+\frac{1}{2} \sum_{j=1}^{n} \beta_{j}\left\|P_{Q_{j}}(A x)-A x\right\|^{2}, \quad \alpha_{i}, \beta_{j}>0,
$$

when combined with (1.5), led to a class of simultaneous projection algorithms which weakly converge to a minimizer of $f$ over $C$. The above $f$ has been introduced in [CEKB05] in the Euclidean space and further considered in [Xu06] and [MR07] in a general Hilbert space. It can be shown that $\nabla f$ is Lipschitz continuous with $L=\sum_{i=1}^{m} \alpha_{i}+\|A\|^{2} \sum_{j=1}^{n} \beta_{j}$.

On the other hand, by the Lipschitz continuity of $\nabla f$ and with a fixed value of $\lambda_{k}=\lambda \in(0,2)$, the PGM method (1.5) becomes an example of the well-known Krasnosel'skiu-Mann [Man53, Kra55] method with an averaged operator. Thanks to the above observation, the weak convergence of the $C Q$-method in a Hilbert space was established, for example, in [Byr04] and in [Xu10]. See also [Xu11] for a weak convergence result with varying $\lambda_{k} \in[\varepsilon, 2-\varepsilon]$ in the PGM.

In some cases, it may happen that one has more information regarding the sets $C$ and $Q$ which determine the SCFP (1.1). For example, following Yang [Yan04], one could assume that

$$
C:=\left\{x \in \mathcal{H}_{1} \mid c(x) \leq 0\right\} \quad \text { and } \quad Q:=\left\{y \in \mathcal{H}_{2} \mid q(y) \leq 0\right\}
$$

for some lower semi-continuous convex functions $c: \mathcal{H}_{1} \rightarrow \mathbb{R}$ and $q: \mathcal{H}_{2} \rightarrow \mathbb{R}$ and consider a subgradient variant of the $C Q$-method

$$
x_{0} \in \mathcal{H}_{1} ; \quad x_{k+1}:=P_{c}\left(x_{k}+\frac{\lambda_{k}}{\|A\|^{2}} A^{*}\left(P_{q}\left(A x_{k}\right)-A x_{k}\right)\right), \quad k=0,1,2, \ldots,
$$

where both metric projections $P_{C}$ and $P_{Q}$ were formally replaced by the corresponding subgradient projections $P_{c}$ and $P_{q}$; see Example 2.4 for a precise definition. Yang [Yan04] shows that method (1.9) converges to some point in the solution set $C \cap A^{-1}(Q) \neq \emptyset$ in the finite dimensional setting and when $\lambda_{k}=\lambda \in(0,2)$. A weak convergence result in a Hilbert space was later established by $\mathrm{Xu}$ in [Xu10]. A simultaneous subgradient projection algorithm for the MSSCFP was also considered in [CMS07].

Another, more general, situation may occur in the case of the split common fixed point problem [CS09], where

$$
C:=\text { Fix } S \quad \text { and } \quad Q:=\operatorname{Fix} T
$$

for given operators $S$ and $T$, which are assumed to be cutters; see Definition 2.3. Again, by formally replacing the metric projections $P_{C}$ and $P_{Q}$ in (1.3) by $S$ and $T$, respectively, we arrive at the following fixed-point variant of the $C Q$-method:

$$
x_{0} \in \mathcal{H}_{1} ; \quad x_{k+1}:=S\left(x_{k}+\frac{\lambda_{k}}{\|A\|^{2}} A^{*}\left(T\left(A x_{k}\right)-A x_{k}\right)\right), \quad k=0,1,2, \ldots
$$


Obviously, since the subgradient projection is a cutter (see Example 2.4), method (1.11) extends (1.9). Observe that, in general, (1.11) and (1.9) are neither a variant of the PGM (no differentiability), nor of the Krasnosel'skiu-Mann method because a cutter need not be nonexpansive. Nevertheless, there are several results showing the convergence of method (1.11); see, for example, [CS09] in the finite dimensional setting or [Mou11], [WX11] and [Ceg15] for weak convergence in Hilbert space. The main assumption in the above-mentioned results is the demi-closedness of $\mathrm{Id}-S$ and Id $-T$ at zero, which we call in this paper weak regularity (see Definition 2.9). Note that only in [Ceg15, Example 6.1] the relaxation parameters $\lambda_{k} \in[\varepsilon, 2-\varepsilon]$, whereas $\lambda_{k}=\lambda \in(0,2)$ in [CS09, Mou11, WX11].

Observe that in all of the above $C Q$-type methods, the computation of the next iterate requires knowing the operator norm $\|A\|$ or its estimation; see, for example, [Byr02, Proposition 4.1], where the norm of $A$ was estimated for a sparse matrix $A$. Other solutions can be found, for example, in [QX05] and [Yan05]. One of the simplest and the most elegant one is due to López et al. [LMWX12], who suggested to consider the following variation of the $C Q$-method:

$$
x_{0} \in \mathcal{H}_{1} ; \quad x_{k+1}:=P_{C}\left(x_{k}-\lambda_{k} \frac{2 f\left(x_{k}\right)}{\left\|\nabla f\left(x_{k}\right)\right\|^{2}} \nabla f\left(x_{k}\right)\right), \quad k=0,1,2, \ldots,
$$

with $f$ defined in (1.4). Observe that (1.12) is indeed a $C Q$-type method, which we call here the extrapolated $C Q$-method since, after expanding, it can be explicitly written as

$$
x_{0} \in \mathcal{H}_{1} ; \quad x_{k+1}=P_{C}\left(x_{k}+\frac{\lambda_{k} \tau\left(x_{k}\right)}{\|A\|^{2}} A^{*}\left(P_{Q}\left(A x_{k}\right)-A x_{k}\right)\right), \quad k=0,1,2, \ldots,
$$

with $\tau(x)$ defined by

$$
\tau(x):=\frac{2\|A\|^{2} f(x)}{\|\nabla f(x)\|^{2}}=\left(\frac{\|A\|\left\|P_{Q}(A x)-A x\right\|}{\left\|A^{*}\left(P_{Q}(A x)-A x\right)\right\|}\right)^{2} \geq 1
$$

for $A x \notin Q$ and $\tau(x):=1$ otherwise. Observe that $x_{k+1}$ does not, in fact, depend on $\|A\|$. Weak convergence of method (1.12) was established in [LMWX12] under the assumption that $C \cap A^{-1}(Q) \neq \emptyset$ and $\lambda_{k} \in[\varepsilon, 2-\varepsilon]$. Again, by formally replacing $P_{C}$ and $P_{Q}$ in (1.13) and (1.14) by weakly regular cutters $S$ and $T$, respectively, Cegielski established weak convergence of the above method in [Ceg16].

Recently, Wang et al. [WHLY17] have formulated a sufficient condition for a linear rate of convergence of the extrapolated $C Q$-method (1.13)-(1.14) in terms of bounded linear regularity of the SCFP, that is, when for all $r>0$, there is $\gamma_{r}>0$ such that for all $x \in C \cap B(0, r)$, we have

$$
\gamma_{r} d\left(x, C \cap A^{-1}(Q)\right) \leq d(A x, Q) .
$$

In particular, the above condition holds when $A(C) \cap \operatorname{int} Q \neq \emptyset$; see [WHLY17, Proposition 2.5] for more details. We comment on this condition in connection with our work below; see Remark 6.7.

\section{Contribution of our paper}

Based on the above short overview, one could distinguish between three different approaches to the study of the convergence properties of various $C Q$-type methods. The first one is viewed through the projected gradient method related to a certain objective $f$. The second one is a Krasnosel'skiı̌-Mann approach with a certain averaged mapping. The last one is a more general fixed point approach, where the metric projections $P_{C}$ and $P_{Q}$ are formally replaced by abstract operators $S$ and $T$. In this paper we focus on the latter case.

To this end, we introduce the Landweber transform $\mathcal{L}\{\cdot\}$, which for a given operator $T: \mathcal{H}_{2} \rightarrow \mathcal{H}_{2}$ assigns an operator $\mathcal{L}\{T\}: \mathcal{H}_{1} \rightarrow \mathcal{H}_{1}$ defined by

$$
\mathcal{L}\{T\} x:=x+\frac{1}{\|A\|^{2}} A^{*}(T(A x)-A x), \quad x \in \mathcal{H}_{1},
$$

which we call in this paper the Landweber operator corresponding to $T$. Observe that we can rewrite (1.3), (1.9) and (1.11) by using $\mathcal{L}\left\{P_{Q}\right\}, \mathcal{L}\left\{P_{q}\right\}$ and $\mathcal{L}\{T\}$, respectively. Moreover, one can show that in all of the above-mentioned cases, we have

$$
A^{-1}(Q)=\text { Fix } \mathcal{L}\left\{P_{Q}\right\}=\text { Fix } \mathcal{L}\left\{P_{q}\right\}=\text { Fix } \mathcal{L}\{T\} ;
$$


see [Ceg15]. Thus the abstract study of the Landweber transform may indeed contribute to the convergence analysis of various $C Q$-type methods.

The main purpose of this paper is to examine which properties of $T$ can be preserved by the Landweber transform. In particular, it is known that the Landweber transform of a (firmly/quasi) nonexpansive operator is again (firmly/quasi) nonexpansive (see [WX11, Lemma 3.1], [Ceg15, Lemma 4.1] and [Ceg16, Proposition 4]). Moreover, the transform of a weakly/boundedly regular operator preserves the type of the regularity; see [WX11, proof of Thm. 3.3] and [Ceg15, Lemma 4.1] for the weak regularity, and [CM16, Thm. 4.2] for the bounded regularity under a compact operator $A$ with closed range.

The main contribution of this paper is to formulate sufficient conditions which ensure that the Landweber transform preserves bounded linear regularity which, as far as we know, is new. Moreover, we show that compactness of $A$ is no longer needed for the preservation of bounded regularity. For both of these results see Theorem 4.7. In addition, based on (1.13) and (1.14), we consider the extrapolated Landweber operator for which we establish similar results as in the nonextrapolated case.

We would like to emphasize that by knowing the regularities of the operators $S$ and $\mathcal{L}\{T\}$, in view of the recent paper [CRZ18] (see Theorem 2.12), we are able to establish the corresponding weak, norm and linear convergence of $C Q$-type methods without restricting ourselves just to projections. In particular, we formulate sufficient conditions for linear convergence of the subgradient and cutter methods described in (1.9) and (1.11). We comment on this in detail in Section 6.

Finally, in order to formulate the linear rate more explicitly, we investigate in detail the closed range theorem; see Lemma 3.2. To this end we introduce the new quantity $|A|:=\inf \{\|A x\| \mid x \in$ $\left.(\operatorname{ker} A)^{\perp},\|x\|=1\right\}$, which, for a matrix $A$ turns out to be the square root of the smallest positive eigenvalue of the matrix $A^{*} A$. In this connection, recall that the spectral norm of the matrix $A$ is the square root of the largest eigenvalue of $A^{*} A$. In addition, we show that, similarly to the properties of $\|A\|$, we have $|A|=\left|A^{*}\right|=\sqrt{\left|A A^{*}\right|}=\sqrt{\left|A^{*} A\right|}$.

\section{Organization of our paper}

In Section 2 we recall several necessary tools which are used in the establishing our main results. In Section 3 we present the closed range theorem. In Section 4 we formally introduce the Landweber transform and investigate its properties. In Section 5 we adjust the results from the previous section to the extrapolated Landweber operator. Finally, in Section 6 we present a few convergence results for various $C Q$-type methods.

\section{Preliminaries}

Let $\mathcal{H}$ be a real Hilbert space. We divide the preliminaries into three separate subsections.

\subsection{Fejér monotone sequences}

Definition 2.1 Let $F \subseteq \mathcal{H}$ be a nonempty, closed and convex set, and let $\left\{x_{k}\right\}_{k=0}^{\infty}$ be a sequence in $\mathcal{H}$. We say that $\left\{x_{k}\right\}_{k=0}^{\infty}$ is Fejér monotone with respect to $F$ if

$$
\left\|x_{k+1}-z\right\| \leq\left\|x_{k}-z\right\|
$$

for all $z \in F$ and every integer $k=0,1,2, \ldots$

Theorem 2.2 Let the sequence $\left\{x_{k}\right\}_{k=0}^{\infty} \subseteq \mathcal{H}$ be Fejér monotone with respect to $F$. Then

(i) $\left\{x_{k}\right\}_{k=0}^{\infty}$ converges weakly to some point $x^{\infty} \in F$ if and only if all its weak cluster points lie in $F$.

(ii) $\left\{x_{k}\right\}_{k=0}^{\infty}$ converges strongly to some point $x^{\infty} \in F$ if and only if $d\left(x_{k}, F\right) \rightarrow 0$.

(iii) If there is some constant $q \in(0,1)$ such that $d\left(x_{k+1}, F\right) \leq q d\left(x_{k}, F\right)$ holds for every $k=0,1,2, \ldots$, then $\left\|x_{k}-x^{\infty}\right\| \leq 2 d\left(x_{0}, F\right) q^{k}$ for some $x_{\infty} \in F$.

Proof. See, for example, [BB96, Theorem 2.16 and Proposition 1.6]. 


\subsection{Quasi-nonexpansive Operators}

Definition 2.3 Let $T: \mathcal{H} \rightarrow \mathcal{H}$ be an operator with a fixed point, that is, Fix $T \neq \emptyset$. We say that $T$ is

(i) quasi-nonexpansive (QNE) if for all $x \in \mathcal{H}$ and all $z \in \operatorname{Fix} T$,

$$
\|T x-z\| \leq\|x-z\| ;
$$

(ii) $\rho$-strongly quasi-nonexpansive ( $\rho$-SQNE), where $\rho \geq 0$, if for all $x \in \mathcal{H}$ and all $z \in$ Fix $U$,

$$
\|T x-z\|^{2} \leq\|x-z\|^{2}-\rho\|T x-x\|^{2} ;
$$

(iii) a cutter if for all $x \in \mathcal{H}$ and all $z \in \operatorname{Fix} T$,

$$
\langle z-T x, x-T x\rangle \leq 0 .
$$

Example 2.4 (Subgradient Projection) Let $f: \mathcal{H} \rightarrow \mathbb{R}$ be a lower semi-continuous and convex function with nonempty sublevel set $S(f, 0):=\{x \in \mathcal{H} \mid f(x) \leq 0\} \neq \emptyset$. For each $x \in \mathcal{H}$, let $g_{f}(x)$ be a chosen subgradient from the subdifferential set $\partial f(x):=\{g \in \mathcal{H} \mid f(y) \geq f(x)+\langle g, y-x\rangle$, for all $y \in \mathcal{H}\}$, which, by [BC17, Proposition 16.27], is nonempty. We call the operator $P_{f}: \mathcal{H} \rightarrow \mathcal{H}$ defined by

$$
P_{f}(x):= \begin{cases}x-\frac{f(x)}{\left\|g_{f}(x)\right\|^{2}} g_{f}(x), & \text { if } f(x)>0 \\ x, & \text { otherwise. }\end{cases}
$$

a subgradient projection related to $f$. It is not difficult to see that $P_{f}$ is a cutter and Fix $P_{f}=S(f, 0)$ [Ceg12, Corollary 4.2.6].

For a given relaxation function $\alpha: \mathcal{H} \rightarrow(0, \infty)$ and an operator $T: \mathcal{H} \rightarrow \mathcal{H}$, we denote by $T_{\alpha}$ the generalized $\alpha(\cdot)$-relaxation of $T$ defined by

$$
T_{\alpha} x:=x+\alpha(x)(T x-x) .
$$

If $\alpha(x)=\alpha$, for some $\alpha>0$, then we simply call $T_{\alpha}$ an $\alpha$-relaxation. In this paper we will consider the former case in the context of extrapolation, where $\alpha(x) \geq 1$; see Section 5 .

Lemma 2.5 Let $T: \mathcal{H} \rightarrow \mathcal{H}$ be an operator with $\operatorname{Fix} T \neq \emptyset$, let $\alpha: \mathcal{H} \rightarrow(0, \infty)$ and $\rho \geq 0$. The following conditions are equivalent:

(i) $T$ is $\rho$-SQNE.

(ii) $T_{\frac{\rho+1}{2}}$ is a cutter.

(iii) For all $x \in \mathcal{H}$ and all $z \in \operatorname{Fix} T$, we have

$$
\langle T x-x, z-x\rangle \geq \frac{\rho+1}{2}\|T x-x\|^{2} .
$$

(iv) For all $x \in \mathcal{H}$ and all $z \in \operatorname{Fix} T$, we have

$$
\left\|T_{\alpha} x-z\right\|^{2} \leq\|x-z\|^{2}-\left(\frac{\rho}{\alpha(x)}+\frac{1-\alpha(x)}{\alpha(x)}\right)\left\|T_{\alpha} x-x\right\|^{2} .
$$

Proof. See [Ceg12, Corollary 2.1.43 and Remark 2.1.31] for the equivalences between (i), (ii) and (iii). The implication (i) $\Rightarrow$ (iv) can be found in [BKRZ18, Corollary 2.3 ]. Actually, slightly adjusting the proof in [BKRZ18], one can deduce that the reverse implication is also true.

Theorem 2.6 Let $U_{i}: \mathcal{H} \rightarrow \mathcal{H}$ be $\rho_{i}$-SQNE, $i=1, \ldots, m$. Define the product operator $P:=\prod_{i=1}^{m} U_{i}:=$ $U_{m} U_{m-1} \ldots U_{1}$. Assume that $\rho:=\min _{i} \rho_{i}>0$ and $F:=\bigcap_{i=1}^{m} \operatorname{Fix} U_{i} \neq \emptyset$. Then $P$ is $(\rho / m)-S Q N E$ and $F=$ Fix $P$.

Proof. See [Ceg12, Theorem 2.1.48]. 


\subsection{Regular Families of Sets and Regular Operators}

The following definition can be found, for example, in [BB96, Definition 5.1] and [BNP15, Definition 5.7].

Definition 2.7 (Regular Sets) Let $S \subseteq \mathcal{H}, C_{i} \subseteq \mathcal{H}, i \in I:=\{1, \ldots, m\}$, be closed and convex with $C:=\bigcap_{i \in I} C_{i} \neq \emptyset$ and let $\mathcal{C}:=\left\{C_{i} \mid i \in I\right\}$. We say that the family $\mathcal{C}$ is

(i) regular over $S$ if for any sequence $\left\{x_{k}\right\}_{k=0}^{\infty} \subseteq S$, we have

$$
\lim _{k \rightarrow \infty} \max _{i \in I} d\left(x_{k}, C_{i}\right)=0 \Longrightarrow \lim _{k \rightarrow \infty} d\left(x_{k}, C\right)=0
$$

(ii) linearly regular over $S$ if there is $\kappa_{S}>0$ such that for every $x \in S$, we have

$$
d(x, C) \leq \kappa_{S} \max _{i \in I} d\left(x, C_{i}\right) .
$$

The constant $\kappa_{S}$ is called a modulus of the linear regularity of $\mathcal{C}$ over $S$.

If any of the above regularity conditions holds for every subset $S \subseteq \mathcal{H}$, then we simply omit the phrase "over $S$ ". If the same condition holds when restricted to bounded subsets $S \subseteq \mathcal{H}$, then we precede the term with the adverb boundedly.

Below we list a few known examples of regular families of sets. For an extended list, see [BB96] or [BNP15].

Example 2.8 Let $C_{i} \subseteq \mathcal{H}, i \in I:=\{1, \ldots, m\}$, be closed and convex with $C:=\bigcap_{i \in I} C_{i} \neq \emptyset$, and let $\mathcal{C}:=\left\{C_{i} \mid i \in I\right\}$.

(i) If $\operatorname{dim} \mathcal{H}<\infty$, then $\mathcal{C}$ is boundedly regular;

(ii) If all $C_{i}, i \in I$, are half-spaces, then $\mathcal{C}$ is linearly regular;

(iii) If $C_{1} \cap \operatorname{int}\left(\bigcap_{i=2}^{m} C_{i}\right) \neq \emptyset$, then $\mathcal{C}$ is boundedly linearly regular;

(iv) If $\operatorname{dim} \mathcal{H}<\infty, C_{i}$ is a half-space, $i=1, \ldots, p$, and $\bigcap_{i=1}^{p} C_{i} \cap \bigcap_{i=p+1}^{m} \operatorname{ri} C_{i} \neq \emptyset$, then $\mathcal{C}$ is boundedly linearly regular.

The following definition was introduced in [CRZ18, Definitions 3.1 and 4.1] (see also references therein for similar concepts).

Definition 2.9 (Regular Operators) Let $\left\{T_{k}\right\}_{k=0}^{\infty}$ be a sequence of operators $T_{k}: \mathcal{H} \rightarrow \mathcal{H}$ with $F:=$ $\bigcap_{k=0}^{\infty}$ Fix $T_{k} \neq \emptyset$ and let $S \subseteq \mathcal{H}$ be nonempty. We say that $\left\{T_{k}\right\}_{k=0}^{\infty}$ is

(i) weakly regular over $S$ if for any sequence $\left\{x_{k}\right\}_{k=0}^{\infty} \subseteq S$ and for any point $x_{\infty} \in \mathcal{H}$, we have

$$
\left.\begin{array}{l}
x_{n_{k}} \rightarrow x_{\infty} \\
T_{k} x_{k}-x_{k} \rightarrow 0
\end{array}\right\} \quad \Longrightarrow \quad x_{\infty} \in F
$$

(ii) regular over $S$ if for any sequence $\left\{x_{k}\right\}_{k=0}^{\infty} \subseteq S$, we have

$$
\lim _{k \rightarrow \infty}\left\|T_{k} x_{k}-x_{k}\right\|=0 \Longrightarrow \lim _{k \rightarrow \infty} d\left(x_{k}, F\right)=0
$$

(iii) linearly regular over $S$ if there is $\delta_{S}>0$ such that for every point $x \in S$, we have

$$
\left\|T_{k} x-x\right\| \geq \delta_{S} d(x, F) .
$$

The constant $\delta_{S}$ is called a modulus of the linear regularity of $T$ over $S$. 
If any of the above regularity conditions holds for every subset $S \subseteq \mathcal{H}$, then we simply omit the phrase "over $S$ ". If the same condition holds when restricted to bounded subsets $S \subseteq \mathcal{H}$, then we precede the term with the adverb boundedly. Since there is no need to distinguish between boundedly weakly and weakly regular operators, we call both weakly regular. If any of the above regularities holds for a constant sequence with $T_{k}=T$ for some $T$, then we simply refer to the regularity of the operator $T$ defined by the corresponding regularity of the sequence $\{T\}_{k=0}^{\infty}$.

Obviously the metric projection is linearly regular and thus (weakly) regular. The example below shows that in some cases the subgradient projection has similar properties. The proof of part (i) can be found, for example, in [Ceg12, Theorem 4.2.7]). The proof of part (iii) in the finite dimensional setting can be found in [CRZ18, Example 3.5 (iii)], which we extend here to the infinite dimensional case.

Example 2.10 (Regularity of Subgradient Projection) Let $P_{f}$ be a subgradient projection as defined in Example 2.4. Assume that $\partial f$ is uniformly bounded on bounded sets (see Remark 2.11). Then the following statements hold:

(i) $P_{f}$ is weakly regular.

(ii) If $f$ is $\alpha$-strongly convex, where $\alpha>0$, then $P_{f}$ is boundedly regular.

(iii) If $f(z)<0$ for some $z$, then $P_{f}$ is boundedly linearly regular.

In particular, if $\mathcal{H}$ is finite dimensional, then $\partial f$ is uniformly bounded on bounded sets and by the equivalence between weak and strong convergence, $P_{f}$ is boundedly regular.

Proof. Let $B(z, r)$ be a ball with center $z \in S(f, 0)$ and radius $r>0$. In order to show the weak/bounded/bounded linear regularity of $P_{f}$, it suffices to show the corresponding regularity over $B(z, r)$ for arbitrary $r>0$.

Before proceeding, let us observe that for any $x \in S(f, 0)$, we have

$$
0=f_{+}(x)=\left\|P_{f} x-x\right\|=d(x, S(f, 0)) .
$$

Moreover, by assumption, for any $r>0$, there is $M>0$ such that $\|g(x)\| \leq M$ for all $x \in B(z, r)$ and all $g(x) \in \partial f(x)$. Hence, for all $x \in B(z, r)$ such that $f(x)>0$, we have

$$
\left\|P_{f} x-x\right\| \geq \frac{f(x)}{\|g(x)\|} \geq \frac{f_{+}(x)}{M} .
$$

Part (i). Let $r>0$ be arbitrary. Assume that $B(z, r) \ni x_{n_{k}} \rightarrow x_{\infty}$ and $\left\|P_{f} x_{k}-x_{k}\right\| \rightarrow 0$. By combining (2.12) and (2.13), we get $f_{+}\left(x_{k}\right) \rightarrow 0$. Since a lower semi-continuous and convex function is weakly lower semi-continuous [BC17, Theorem 9.1], we have $0=\liminf _{k} f_{+}\left(x_{n_{k}}\right) \geq \liminf _{k} f\left(x_{n_{k}}\right) \geq$ $f\left(x_{\infty}\right)$, that is, $x_{\infty} \in$ Fix $P_{f}$. This shows that $P_{f}$ is weakly regular over $B(z, r)$.

Part (ii). Let $r>0$ be arbitrary. Recall that $f$ is $\alpha$-strongly convex if

$$
f(\lambda x+(1-\lambda) y) \leq \lambda f(x)+(1-\lambda) f(y)-\frac{\alpha}{2} \lambda(1-\lambda)\|x-y\|^{2}
$$

for all $x, y \in \mathcal{H}$ and $\lambda \in[0,1]$. In particular, for any $x \notin S(f, 0)$, by setting $y=P_{S(f, 0)} x$ and $\lambda=\frac{1}{2}$, we have

$$
f_{+}(x)=f(x) \geq 2 \underbrace{f\left(\frac{1}{2} x+\frac{1}{2} P_{S(f, 0)} x\right)}_{>0}-\underbrace{f\left(P_{S(f, 0)} x\right)}_{=0}+\frac{\alpha}{4}\left\|x-P_{S(f, 0)} x\right\|^{2} \geq \frac{\alpha}{4} d^{2}(x, S(f, 0)) .
$$

This, when combined with (2.12) and (2.13), easily leads to the regularity of $P_{f}$ over $B(z, r)$.

Part (iii). Assume that $f(z)<0$ and let $x \in B(z, r)$ be such that $f(x)>0$. Define $y:=\lambda z+(1-\lambda) x$, where $\lambda:=\frac{f(x)}{f(x)-f(z)}>0$. Then $y \in S(f, 0)$ and, by the definition of the metric projection, we have

$$
d(x, S(f, 0)) \leq\|x-y\|=\lambda\|x-z\| \leq \frac{f_{+}(x)}{-f(z)} r .
$$

The inequality above, when combined with (2.12) and (2.13), leads to linear regularity of $P_{f}$ over $B(z, r)$. 
Remark 2.11 Let $f: \mathcal{H} \rightarrow \mathbb{R}$ be lower semi-continuous and convex. We recall that $\partial f$ is uniformly bounded on bounded sets if and only if $f$ is Lipschitz continuous on bounded sets, a condition which holds if and only if $f$ maps bounded sets onto bounded sets; see, for example, [BB96, Proposition 7.8]. The above holds true, in particular, when $\mathcal{H}=\mathbb{R}^{n}$.

The theorem below summarizes some of the properties of regular operators discussed in [CRZ18]. We apply this theorem in Section 6 only for two sequences while studying the convergence of various $C Q$-type methods.

Theorem 2.12 Let $\left\{U_{1}^{k}\right\}_{k=0}^{\infty}, \ldots,\left\{U_{m}^{k}\right\}_{k=0}^{\infty}$ be given sequences of $\rho_{i}^{k}$-SQNE operators $U_{i}^{k}: \mathcal{H} \rightarrow \mathcal{H}$ with Fix $U_{i}^{k}=F_{i}, i=1,2, \ldots, m$, (in particular each sequence can be a constant consisting only of one operator $\left.U_{i}\right)$. Define the product operator $P_{k}$ by

$$
P_{k}:=\prod_{i=1}^{m} U_{i}^{k}
$$

Assume that $\rho:=\inf _{i, k} \rho_{i}^{k}>0$ and $F:=\bigcap_{i=1}^{m} F_{i} \neq \emptyset\left(P_{k}\right.$ is $(\rho / m)$-SQNE and $F=$ Fix $P_{k}$ by Theorem 2.6). Let $B=B(z, r)$ for some $z \in F$ and $r>0$. Then the following statements hold:

(i) If for each $i=1, \ldots, m$, the sequence $\left\{U_{i}^{k}\right\}_{k=0}^{\infty}$ is weakly regular over $B$, then $\left\{P_{k}\right\}_{k=0}^{\infty}$ is also weakly regular over $B$.

(ii) If for each $i=1, \ldots, m$, the sequence $\left\{U_{i}^{k}\right\}_{k=0}^{\infty}$ is regular over $B$ and the family of sets $\left\{F_{1}, \ldots, F_{m}\right\}$ is regular over $B$, then $\left\{P_{k}\right\}_{k=0}^{\infty}$ is also regular over $B$.

(iii) If for each $i=1, \ldots, m$, the sequence $\left\{U_{i}^{k}\right\}_{k=0}^{\infty}$ is linearly regular over $B$ with modulus $\delta_{i}$ and the family of sets $\left\{F_{1}, \ldots, F_{m}\right\}$ is linearly regular over $B$ with modulus $\kappa>0$, then $\left\{P_{k}\right\}_{k=0}^{\infty}$ is also linearly regular over $B$ with modulus

$$
\delta_{P}=\frac{\rho \delta^{2}}{2 m \kappa^{2}}
$$

where $\delta:=\min _{i} \delta_{i}$.

Proof. This theorem is a particular case of [CRZ18, Corollary 5.5].

Remark 2.13 (Erratum to [CRZ18]) Note that in contrast to Definition 2.9(iii), in [CRZ18] the constant $\delta_{S}^{-1}$ was called a modulus of the linear regularity of $T$ over $S$. Consequently, in all the results of Section 5 in [CRZ18] one should use " $\delta:=\max _{i} \delta_{i}$ " instead of " $\delta:=\min _{i} \delta_{i}$ ". The statement of Theorem 2.12 corrects this unfortunate misprint.

\section{Closed Range Theorem}

Let $\mathcal{H}_{1}$ and $\mathcal{H}_{2}$ be two Hilbert spaces, $A: \mathcal{H}_{1} \rightarrow \mathcal{H}_{2}$ be a nonzero bounded linear operator and $A^{*}$ : $\mathcal{H}_{2} \rightarrow \mathcal{H}_{1}$ be its adjoint operator defined by

$$
\langle A x, y\rangle=\left\langle x, A^{*} y\right\rangle \quad \text { for all } x \in \mathcal{H}_{1} \text { and all } y \in \mathcal{H}_{2} .
$$

We denote: $\operatorname{ker} A:=\left\{x \in \mathcal{H}_{1} \mid A x=0\right\}$ - the kernel (null space) of $A$, im $A:=\left\{y \in \mathcal{H}_{2} \mid A x=y\right.$ for some $\left.x \in \mathcal{H}_{1}\right\}$ - the image (range) of $A, \operatorname{cl} C$ - the closure of a subset $C \subseteq \mathcal{H}_{1}, V^{\perp}:=\left\{y \in \mathcal{H}_{1} \mid\langle x, y\rangle=0\right.$ for all $x \in V\}$ - the orthogonal complement of a subspace $V \subseteq \mathcal{H}_{1}$. It is not difficult to see that

$$
\operatorname{ker} A=\operatorname{ker} A^{*} A \quad \text { and } \quad \operatorname{ker} A^{*}=\operatorname{ker} A A^{*} .
$$

Moreover, it is well known that

$$
(\operatorname{ker} A)^{\perp}=\operatorname{cl}\left(\operatorname{im} A^{*}\right) \quad \text { and } \quad\left(\operatorname{ker} A^{*}\right)^{\perp}=\operatorname{cl}(\operatorname{im} A) ;
$$

see, for example, [Deu01, Lemma 8.33]. In what follows the following property turns out to be useful. 
Proposition 3.1 The operator $A^{\#}:(\operatorname{ker} A)^{\perp} \rightarrow \operatorname{im} A$ defined by $A^{\#}:=\left.A\right|_{(\operatorname{ker} A)^{\perp}}$ is a bijection.

Proof. To see that $A^{\#}$ is surjective, let $y \in \operatorname{im} A$ and $x \in \mathcal{H}_{1}$ be such that $y=A x$. Then $x=x^{\prime}+x^{\prime \prime}$ with $x^{\prime} \in \operatorname{ker} A$ and $x^{\prime \prime} \in(\operatorname{ker} A)^{\perp}$. We have $y=A x=A\left(x^{\prime}+x^{\prime \prime}\right)=A x^{\prime \prime}=A^{\#} x^{\prime \prime}$, which proves that $\operatorname{im} A^{\#}=\operatorname{im} A$. To see that $A^{\#}$ is injective, assume that $A^{\#} x_{1}=A^{\#} x_{2}$ for some $x_{1}, x_{2} \in(\operatorname{ker} A)^{\perp}$. Then $A^{\#}\left(x_{1}-x_{2}\right)=A\left(x_{1}-x_{2}\right)=0$, that is, $x_{1}-x_{2} \in \operatorname{ker} A$. Since we also have $x_{1}-x_{2} \in(\operatorname{ker} A)^{\perp}$ and ker $A \cap(\operatorname{ker} A)^{\perp}=\{0\}$, it follows that $x_{1}=x_{2}$. This proves that $A^{\#}$ is a bijection.

By $\|A\|:=\sup \left\{\|A x\| \mid x \in \mathcal{H}_{1},\|x\|=1\right\}$ we denote the norm of the operator $A$. It is not difficult to see that the norm of $A$ satisfies

$$
\|A\|=\sup \left\{\|A x\| \mid x \in(\operatorname{ker} A)^{\perp},\|x\|=1\right\} .
$$

Moreover,

$$
\|A\|=\left\|A^{*}\right\|=\sqrt{\left\|A^{*} A\right\|}=\sqrt{\left\|A A^{*}\right\|} ;
$$

see, for example, [Deu01, Theorem 8.25]. Analogously, we define

$$
|A|:=\inf \left\{\|A x\| \mid x \in(\operatorname{ker} A)^{\perp},\|x\|=1\right\} .
$$

Clearly, for any $x \in(\operatorname{ker} A)^{\perp}$, we have

$$
|A| \cdot\|x\| \leq\|A x\| \leq\|A\| \cdot\|x\|,
$$

where $|A|$ and $\|A\|$ are the largest and the smallest constants, respectively, for which the above inequalities hold. The following lemma provides a list of equivalent conditions for $|A|$ to be greater than zero. The equivalence (i) $\Leftrightarrow(\mathrm{iv})$ is known as the closed range theorem [Yos78, Chapter VII, Section 5].

Lemma 3.2 (Closed Range Theorem) Let $A: \mathcal{H}_{1} \rightarrow \mathcal{H}_{2}$ be a nonzero bounded linear operator. The following statements are equivalent:

(i) $\operatorname{im} A$ is closed;

(iv) $\operatorname{im} A^{*}$ is closed;

(vii) $\operatorname{im} A A^{*}$ is closed;

(x) $\operatorname{im} A^{*} A$ is closed;
(ii) $\operatorname{im} A=\left(\operatorname{ker} A^{*}\right)^{\perp}$;
(iii) $|A|>0$;
(v) $\operatorname{im} A^{*}=(\operatorname{ker} A)^{\perp}$;
(vi) $\left|A^{*}\right|>0$;
(viii) $\operatorname{im} A A^{*}=\left(\operatorname{ker} A A^{*}\right)^{\perp}$;
(ix) $\left|A A^{*}\right|>0$;
(xi) $\operatorname{im} A^{*} A=\left(\operatorname{ker} A^{*} A\right)^{\perp}$;
(xii) $\left|A^{*} A\right|>0$.

Moreover, if any of the above conditions holds, then (compare with (3.1))

$$
\operatorname{im} A A^{*}=\operatorname{im} A \quad \text { and } \quad \operatorname{im} A^{*} A=\operatorname{im} A^{*} .
$$

Furthermore, in this case $A^{\#}$ has a bounded inverse and (compare with (3.4))

$$
|A|=\left|A^{*}\right|=\sqrt{\left|A A^{*}\right|}=\sqrt{\left|A^{*} A\right|}=\frac{1}{\left\|\left(A^{\#}\right)^{-1}\right\|} .
$$

Proof. Step 1. We begin the proof by showing that conditions (i)-(xii) are indeed equivalent.

The equivalence (i) $\Leftrightarrow$ (ii) follows from (3.2) and the fact that the orthogonal complement of a nonempty set in a Hilbert space is a closed subspace; see, for example, [Deu01, Theorem 4.5]. For the equivalence (i) $\Leftrightarrow($ iii), see [Deu01, Theorem 8.18]. Hence, we have obtained the equivalences (i) $\Leftrightarrow($ ii $) \Leftrightarrow($ iii). By replacing $A$ with $A^{*}, A A^{*}$ and $A^{*} A$ in the latter equivalence, we obtain (iv) $\Leftrightarrow(\mathrm{v}) \Leftrightarrow($ vi), (vii) $\Leftrightarrow($ viii) $\Leftrightarrow($ ix) and $(\mathrm{x}) \Leftrightarrow(\mathrm{xi}) \Leftrightarrow(\mathrm{xii})$, respectively. For the equivalence of statements (i) and (iv), see [Yos78, Chapter VII, Section 5]. Thus statements (i)-(vi) are equivalent. Next we show that (iv) $\Leftrightarrow$ (vii). Indeed, assume that $\operatorname{im} A^{*}$ is closed. Then, the equality $\operatorname{im} A^{*}=(\operatorname{ker} A)^{\perp}$ and the orthogonal decomposition theorem yield

$$
\operatorname{im}\left(A A^{*}\right)=A\left(\operatorname{im} A^{*}\right)=A\left((\operatorname{ker} A)^{\perp}\right)=A\left((\operatorname{ker} A)^{\perp} \oplus \operatorname{ker} A\right)=A\left(\mathcal{H}_{1}\right)=\operatorname{im} A,
$$


which is closed by the equivalence between (i) and (iv). This proves that (iv) $\Rightarrow$ (vii). Now assume that $\operatorname{im} A A^{*}$ is closed. We prove that $\operatorname{im} A$ and, consequently, im $A^{*}$ are closed. Indeed, by (3.1) and (3.2), we have

$$
\operatorname{im} A \subseteq \operatorname{cl}(\operatorname{im} A)=\left(\operatorname{ker} A^{*}\right)^{\perp}=\left(\operatorname{ker} A A^{*}\right)^{\perp}=\operatorname{im} A A^{*} \subseteq \operatorname{im} A .
$$

Thus, $\operatorname{cl}(\operatorname{im} A)=\operatorname{im} A$. By interchanging $A$ with $A^{*}$ in the above argument, we can show that $\operatorname{im} A^{*} A=$ $\operatorname{im} A^{*}$ and that (i) $\Leftrightarrow(\mathrm{x})$. Consequently, we have established the equivalence of all the conditions (i)-(xii) and proved (3.7). In the remaining part of the proof we suppose that any of the equivalent conditions (i)-(xii) is satisfied.

Step 2. Suppose that $\operatorname{im} A$ is closed. Then $\operatorname{im} A=\left(\operatorname{ker} A^{*}\right)^{\perp}$. This means that $A^{\#}$ has closed image, that is, $\operatorname{im} A^{\#}=\operatorname{im} A=\left(\operatorname{ker} A^{*}\right)^{\perp}$. This and the fact that $A^{\#}$ is a bijection yield that $A^{\#}$ has a bounded inverse $\left(A^{\#}\right)^{-1}:\left(\operatorname{ker} A^{*}\right)^{\perp} \rightarrow(\operatorname{ker} A)^{\perp}$ (see [Deu01, Theorem 8.19]).

Step 3. By assumption, $\operatorname{im} A^{*}$ is closed, that is, $\operatorname{im} A^{*}=(\operatorname{ker} A)^{\perp}$. Similarly to the definition of $A^{\#}$, we can define the operator $A^{b}:\left(\operatorname{ker} A^{*}\right)^{\perp} \rightarrow(\operatorname{ker} A)^{\perp}$ by $A^{b}:=\left.A^{*}\right|_{\left(\operatorname{ker} A^{*}\right)^{\perp}}$. Moreover, similarly to Proposition 3.1 and Step 2 we can prove that $A^{b}$ is a bijection and has a bounded inverse $\left(A^{b}\right)^{-1}$ : $(\operatorname{ker} A)^{\perp} \rightarrow\left(\operatorname{ker} A^{*}\right)^{\perp}$. Finally, for any $x \in(\operatorname{ker} A)^{\perp}$ and $y \in\left(\operatorname{ker} A^{*}\right)^{\perp}$, we have

$$
\left\langle A^{\#} x, y\right\rangle=\langle A x, y\rangle=\left\langle x, A^{*} y\right\rangle=\left\langle x, A^{b} y\right\rangle
$$

which means that

$$
A^{b}=\left(A^{\#}\right)^{*} \quad \text { and } \quad A^{\#}=\left(A^{b}\right)^{*} .
$$

By [Deu01, Theorem 8.31], we have

$$
\left(A^{b}\right)^{-1}=\left(\left(A^{\#}\right)^{*}\right)^{-1}=\left(\left(A^{\#}\right)^{-1}\right)^{*} .
$$

Step 4. Now we show that (3.8) indeed holds. For arbitrary norm-one $y \in \operatorname{im} A$ and $x \in(\operatorname{ker} A)^{\perp}$ with $A^{\#} x=y$, the first inequality in (3.6) implies that

$$
\left\|\left(A^{\#}\right)^{-1} y\right\|=\left\|\left(A^{\#}\right)^{-1} A^{\#} x\right\|=\|x\| \leq|A|^{-1}\|A x\|=|A|^{-1}\|y\|=|A|^{-1} .
$$

Thus

$$
\left\|\left(A^{\#}\right)^{-1}\right\|=\sup _{\substack{y \in \operatorname{im} A \\\|y\|=1}}\left\|\left(A^{\#}\right)^{-1} y\right\| \leq|A|^{-1}
$$

and $|A| \leq\left\|\left(A^{\#}\right)^{-1}\right\|^{-1}$. We show that $|A| \geq\left\|\left(A^{\#}\right)^{-1}\right\|^{-1}$. Suppose, to the contrary, that $\left\|\left(A^{\#}\right)^{-1}\right\|^{-1}>$ $|A|$. By the definition of $|A|$, for any $k$ there is $x_{k} \in(\operatorname{ker} A)^{\perp}$ with $\left\|x_{k}\right\|=1$ such that

$$
\left\|A^{\#} x_{k}\right\|=\left\|A x_{k}\right\| \leq|A|+\frac{1}{k} .
$$

We have

$$
\begin{aligned}
|A| & <\left\|\left(A^{\#}\right)^{-1}\right\|^{-1}=\left\|\left(A^{\#}\right)^{-1}\right\|^{-1}\left\|x_{k}\right\|=\left\|\left(A^{\#}\right)^{-1}\right\|^{-1}\left\|\left(A^{\#}\right)^{-1} A^{\#} x_{k}\right\| \\
& \leq\left\|\left(A^{\#}\right)^{-1}\right\|^{-1}\left\|\left(A^{\#}\right)^{-1}\right\| \cdot\left\|A^{\#} x_{k}\right\| \leq|A|+\frac{1}{k}
\end{aligned}
$$

which, by letting $k \rightarrow \infty$, leads to a contradiction. Thus

$$
|A|=\frac{1}{\left\|\left(A^{\#}\right)^{-1}\right\|} .
$$

Replacing $A$ with $A^{*}$, we obtain,

$$
\left|A^{*}\right|=\frac{1}{\left\|\left(A^{b}\right)^{-1}\right\|} .
$$

This, when combined with (3.13), implies that

$$
|A|=\frac{1}{\left\|\left(A^{\#}\right)^{-1}\right\|}=\frac{1}{\left\|\left(\left(A^{\#}\right)^{-1}\right)^{*}\right\|}=\frac{1}{\left\|\left(A^{b}\right)^{-1}\right\|}=\left|A^{*}\right|,
$$


which proves a part of (3.8).

Similarly as in Proposition 3.1 and in Step 2, and since $(\operatorname{ker} A)^{\perp}=\left(\operatorname{ker} A^{*} A\right)^{\perp}$, the operator $\left(A^{*} A\right)^{\#}$ : $(\operatorname{ker} A)^{\perp} \rightarrow(\operatorname{ker} A)^{\perp}$, defined by $\left(A^{*} A\right)^{\#}:=\left.A^{*} A\right|_{(\operatorname{ker} A)^{\perp}}$, is a bijection and has a bounded inverse $\left(\left(A^{*} A\right)^{\#}\right)^{-1}$. By [Deu01, Theorem 8.25], we have

$$
\left\|\left(A^{\#}\right)^{-1}\left(\left(A^{\#}\right)^{-1}\right)^{*}\right\|=\left\|\left(A^{\#}\right)^{-1}\right\|^{2} .
$$

Moreover, for $x \in(\operatorname{ker} A)^{\perp}$, we obtain

$$
\left(A^{*} A\right)^{\#} x=A^{*} A x=A^{*} A^{\#} x=A^{\mathrm{b}} A^{\#} x
$$

which, when combined with (3.13) and (3.21), implies that

$$
\left\|\left(\left(A^{*} A\right)^{\#}\right)^{-1}\right\|=\left\|\left(A^{b} A^{\#}\right)^{-1}\right\|=\left\|\left(A^{\#}\right)^{-1}\left(A^{b}\right)^{-1}\right\|=\left\|\left(A^{\#}\right)^{-1}\left(\left(A^{\#}\right)^{-1}\right)^{*}\right\|=\left\|\left(A^{\#}\right)^{-1}\right\|^{2} .
$$

Thus, replacing $A$ with $A^{*} A$ in (3.18), we arrive at

$$
\left|A^{*} A\right|=\frac{1}{\left\|\left(\left(A^{*} A\right)^{\#}\right)^{-1}\right\|}=\frac{1}{\left\|\left(A^{\#}\right)^{-1}\right\|^{2}}=|A|^{2} .
$$

In the same way, one can prove that

$$
\left|A A^{*}\right|=\frac{1}{\left\|\left(A^{b}\right)^{-1}\right\|^{2}}=\left|A^{*}\right|^{2}=|A|^{2} .
$$

This completes the proof of Lemma 3.2.

Remark 3.3 Observe that even if $\operatorname{im} A$ is not closed then, due to the equivalences between (iii), (vi), (ix) and (xii), the equalities from (3.8) will take the following form: $|A|=\left|A^{*}\right|=\sqrt{\left|A A^{*}\right|}=\sqrt{\left|A^{*} A\right|}=0$.

Lemma 3.4 (Compact Operators and Closed Range) Let $A: \mathcal{H}_{1} \rightarrow \mathcal{H}_{2}$ be a nonzero bounded linear operator and assume that $A$ is compact. Let $\Lambda^{+}\left(A^{*} A\right)$ be the set of all positive eigenvalues of $A^{*} A$. Then

$$
\left\|A^{*} A\right\|=\sup \Lambda^{+}\left(A^{*} A\right) \in \Lambda^{+}\left(A^{*} A\right) .
$$

Moreover, we have the following alternative:

(i) The set $\Lambda^{+}\left(A^{*} A\right)$ is finite (for example, when $A$ is a nonzero $m \times n$ matrix). Then

$$
\left|A^{*} A\right|=\min \Lambda^{+}\left(A^{*} A\right)>0
$$

and consequently, the set im $A$ is closed.

(ii) The set $\Lambda^{+}\left(A^{*} A\right)$ is countably infinite. Then

$$
\left|A^{*} A\right|=\inf \Lambda^{+}\left(A^{*} A\right)=0
$$

and consequently, the set $\operatorname{im} A$ is not closed.

Proof. The proof follows from the basic properties of compact self-adjoint operators and the spectral decomposition theorem applied to $A^{*} A$; see, for example [Kre14, Section 15.3] or [DM05, Section 4.8]. We remark in passing that the spectral decomposition theorem is usually presented in the setting of a complex Hilbert space. Nevertheless, one can obtain an analogous result in a real Hilbert space by using, for instance, a complexification argument combined with the fact that all eigenvalues of a self-adjoint operator are real. Hence, when referring to results from [Kre14, Section 15.3] we actually adjust them to the setting of a real Hilbert space.

First observe that since $A$ is compact, the operators $A^{*}$ and consequently $A^{*} A$ are also compact; see [Kre14, Theorem 4.12]. Moreover, since $A^{*} A$ is self-adjoint and positive semi-definite, all of its eigenvalues are nonnegative and, by [Kre14, Theorems 15.11], we indeed have $\left\|A^{*} A\right\|=\sup \Lambda^{+}\left(A^{*} A\right) \in \Lambda^{+}\left(A^{*} A\right)$. By the spectral decomposition theorem [Kre14, Theorem 15.12], we know that $A^{*} A$ has either a finite 
or a countably infinite set of eigenvalues accumulating only at zero, say $\Lambda^{+}\left(A^{*} A\right)=\left\{\lambda_{k} \mid k \in K\right\}$ with $K=\{1, \ldots, N\}$ in the former and $K=\mathbb{N}$ in the latter case. In both cases we may assume that $\lambda_{k}>\lambda_{k+1}$. Moreover, the eigenspaces $E_{k}:=\operatorname{ker}\left(\lambda_{k} \operatorname{Id}-A^{*} A\right)$ corresponding to different eigenvalues are orthogonal and finite dimensional. Finally, for every point $x \in \mathcal{H}_{1}$, we have

$$
x=\sum_{k \in K} P_{k} x+P_{\operatorname{ker} A^{*} A} x \quad \text { and } \quad A^{*} A=\sum_{k \in K} \lambda_{k} P_{k},
$$

where $P_{k}$ is the orthogonal projection of $\mathcal{H}_{1}$ onto the eigenspace $E_{k}$.

Part (i). Assume that $\Lambda^{+}\left(A^{*} A\right)$ is finite. Observe that for any $x \in\left(\operatorname{ker} A^{*} A\right)^{\perp}$, we have $P_{\operatorname{ker}} A^{*} A^{x}=0$. Moreover, since the eigenspaces $E_{k}$ and $E_{l}$ are orthogonal for $k \neq l$, the corresponding projections satisfy $P_{k} P_{l}=P_{l} P_{k}=0$. Using (3.29), we arrive at

$$
\left\|A^{*} A x\right\|^{2}=\left\langle\sum_{k=1}^{N} \lambda_{k} P_{k} x, \sum_{l=1}^{N} \lambda_{l} P_{l} x\right\rangle=\sum_{k=1}^{N} \lambda_{k}^{2}\left\|P_{k} x\right\|^{2} \geq \lambda_{N}^{2} \sum_{k=1}^{N}\left\|P_{k} x\right\|^{2}=\lambda_{N}^{2}\|x\|^{2}
$$

for all $x \in \mathcal{H}_{1}$. Knowing that $\left|A^{*} A\right|$ is the largest number for which the above inequality holds (compare with (3.6)), we obtain $\lambda_{N} \leq\left|A^{*} A\right|$. On the other hand, for any norm-one eigenvector $e_{N} \in E_{N} \subseteq$ $\left(\operatorname{ker} A^{*} A\right)^{\perp}$, we have

$$
\lambda_{N}=\left\|\lambda_{N} e_{N}\right\|=\left\|A^{*} A e_{N}\right\| \geq\left|A^{*} A\right| \cdot\left\|e_{N}\right\|=\left|A^{*} A\right|,
$$

which shows that $\left|A^{*} A\right|=\lambda_{N}$.

Part (ii). Assume that $\Lambda^{+}\left(A^{*} A\right)$ is countably infinite. For each $k=1,2, \ldots$, choose a norm-one eigenvector $e_{k} \in E_{k} \subseteq\left(\operatorname{ker} A^{*} A\right)^{\perp}$. Then we have

$$
\left|A^{*} A\right|=\inf \left\{\left\|A^{*} A x\right\| \mid x \in\left(\operatorname{ker} A^{*} A\right)^{\perp},\|x\|=1\right\} \leq \inf _{k}\left\|A^{*} A e_{k}\right\|=\inf _{k} \lambda_{k}=0,
$$

where the last equality holds because zero is the only possible accumulation point of $\Lambda^{+}\left(A^{*} A\right)$. This completes the proof.

\section{Landweber Operators}

Let $\mathcal{H}_{1}$ and $\mathcal{H}_{2}$ be two Hilbert spaces, let $A: \mathcal{H}_{1} \rightarrow \mathcal{H}_{2}$ be a nonzero bounded linear operator and let $T: \mathcal{H}_{2} \rightarrow \mathcal{H}_{2}$ be an arbitrary operator.

Definition 4.1 The operator $\mathcal{L}\{T\}: \mathcal{H}_{1} \rightarrow \mathcal{H}_{1}$ defined by

$$
\mathcal{L}\{T\} x:=x+\frac{1}{\|A\|^{2}} A^{*}(T(A x)-A x), \quad x \in \mathcal{H}_{1},
$$

is called the Landweber operator ( corresponding to $T$ ). We call the operation $T \mapsto \mathcal{L}\{T\}$ the Landweber transform.

We recall that in the literature the Landweber operator is usually defined for $T=P_{Q}$, where $Q \subseteq \mathcal{H}_{2}$ is closed and convex (see, for example, [Byr02]).

Remark 4.2 Observe that the Landweber transform of the identity on $\mathcal{H}_{2}$ is again the identity, but on $\mathcal{H}_{1}$. This can be written briefly as follows:

$$
\mathcal{L}\{\mathrm{Id}\}=\mathrm{Id} .
$$

Moreover, for given operators $T: \mathcal{H}_{2} \rightarrow \mathcal{H}_{2}$ and $T_{i}: \mathcal{H}_{2} \rightarrow \mathcal{H}_{2}$, weights $\omega_{i} \geq 0, i=1,2, \ldots, m$, with $\sum_{i=1}^{m} \omega_{i}=1$ and a relaxation parameter $\lambda \geq 0$, the Landweber transform satisfies

$$
\mathcal{L}\left\{T_{\lambda}\right\}=\mathcal{L}_{\lambda}\{T\},
$$


where $T_{\lambda}:=\operatorname{Id}+\lambda(T-\mathrm{Id})$ and $\mathcal{L}_{\lambda}\{T\}:=\mathrm{Id}+\lambda(\mathcal{L}\{T\}-\mathrm{Id})$ denote the $\lambda$-relaxation of $T$ and $\mathcal{L}\{T\}$, respectively, and

$$
\mathcal{L}\left\{\sum_{i=1}^{m} \omega_{i} T_{i}\right\}=\sum_{i=1}^{m} \omega_{i} \mathcal{L}\left\{T_{i}\right\} .
$$

Furthermore, if $A$ is unitary (that is, when $A^{*} A=\mathrm{Id}$ ), then $\mathcal{L}\{T\}=A^{*} T A$ and thus

$$
\mathcal{L}\left\{\prod_{i=1}^{m} T_{i}\right\}=\prod_{i=1}^{m} \mathcal{L}\left\{T_{i}\right\} .
$$

In order to formulate our next lemma, we recall [BBR78] that an operator $T: \mathcal{H} \rightarrow \mathcal{H}$ is called $\alpha$-averaged $(\alpha-\mathrm{AV})$, where $\alpha \in(0,1)$, if $T$ is the $\alpha$-relaxation of some nonexpansive operator $U$, that is, $T=(1-\alpha) \operatorname{Id}+\alpha U$.

Lemma 4.3 Let $\mathcal{L}\{T\}$ be the Landweber operator corresponding to $T: \mathcal{H}_{2} \rightarrow \mathcal{H}_{2}, \alpha \in(0,1)$ and $\rho \geq 0$.

(i) If $T$ (firmly) nonexpansive, then $\mathcal{L}\{T\}$ is also (firmly) nonexpansive.

(ii) If $T$ is $\alpha-A V$, then $\mathcal{L}\{T\}$ is also $\alpha-A V$.

(iii) If $T$ is a cutter and $\operatorname{im} A \cap \operatorname{Fix} T \neq \emptyset$, then $\mathcal{L}\{T\}$ is also a cutter and $\operatorname{Fix} \mathcal{L}\{T\}=A^{-1}(\operatorname{Fix} T)$.

(iv) If $T$ is $\rho$-SQNE and $\operatorname{im} A \cap \operatorname{Fix} T \neq \emptyset$, then $\mathcal{L}\{T\}$ is also $\rho$-SQNE and, as in (iii), we have Fix $\mathcal{L}\{T\}=A^{-1}(\operatorname{Fix} T)$.

Proof. (i) If $T$ is firmly nonexpansive, then the result follows from [Ceg16, Proposition 4]. Now suppose that $T$ is nonexpansive, that is, $T=\operatorname{Id}+2(U-\mathrm{Id})$ for some firmly nonexpansive operator $U$; see, for example, [Ceg12, Theorem 2.2.10]. Hence, by (4.3), $\mathcal{L}\{T\}=\mathcal{L}\left\{U_{2}\right\}=\mathcal{L}_{2}\{U\}$, which is nonexpansive. (ii) By [Ceg12, Corollary 2.2.17], $T$ is $\alpha$-AV if and only if $T=\mathrm{Id}+2 \alpha(U-\mathrm{Id})$ for some firmly nonexpansive operator $U$. Hence the result follows from (i) and (4.3). A proof of (iii) can be found in [WX11, Lemma 3.1]. (iv) It follows from Lemma 2.5 that $T$ is $\rho$-SQNE if and only if $T=\operatorname{Id}+\frac{2}{\rho+1}(U-\mathrm{Id})$ for some cutter $U$, which in this case equals $T_{(\rho+1) / 2}$. Hence we can refer, once again, to (4.3) which, when combined with (iii), completes the proof. An independent proof of parts (iii) and (iv) can also be found in [Ceg15, Lemma 4.1].

Observe that the equality Fix $\mathcal{L}\{T\}=A^{-1}(\operatorname{Fix} T)$ yields the following equivalence:

$$
A^{*}(T(A x)-A x)=0 \Longleftrightarrow A x \in \operatorname{Fix} T .
$$

We use this fact later in Section 5 while defining the extrapolated Landweber operator. Before formulating the main result of this section, we prove several auxiliary lemmata.

Lemma 4.4 Let $A: \mathcal{H}_{1} \rightarrow \mathcal{H}_{2}$ be a nonzero bounded linear operator with closed $\operatorname{im} A$ and let $T: \mathcal{H}_{2} \rightarrow \mathcal{H}_{2}$ be quasi-nonexpansive. Assume that $\operatorname{im} A \cap \operatorname{Fix} T \neq \emptyset$. Then for any $x \in \mathcal{H}_{1}$, we have

$$
\frac{1}{\|A\|} d(A x, \operatorname{im} A \cap \operatorname{Fix} T) \leq d(x, \operatorname{Fix} \mathcal{L}\{T\}) \leq \frac{1}{|A|} d(A x, \operatorname{im} A \cap \operatorname{Fix} T) .
$$

Proof. To shorten our notation, denote $F:=\operatorname{im} A \cap$ Fix $T$. Let $x \in \mathcal{H}_{1}$. Clearly, $F$ is closed and convex and $d(A x, F)=\left\|A x-P_{F}(A x)\right\|$. Let $z:=P_{F}(A x)$. By the equality Fix $\mathcal{L}\{T\}=A^{-1}(\operatorname{Fix} T)$ (see Lemma 4.3), there is a point $w \in \operatorname{Fix} \mathcal{L}\{T\}$ such that $A w=z$. Let

$$
u:=x-w=u^{\prime}+u^{\prime \prime},
$$

where, by the orthogonal decomposition theorem, $u^{\prime} \in \operatorname{ker} A$ and $u^{\prime \prime} \in(\operatorname{ker} A)^{\perp}$. Note that

$$
w^{\prime}:=w+u^{\prime} \in \operatorname{Fix} \mathcal{L}\{T\}
$$


because $A w^{\prime}=A w+A u^{\prime}=A w=z \in \operatorname{Fix} T$. Moreover,

$$
x-w^{\prime}=u^{\prime \prime} \in(\operatorname{ker} A)^{\perp} .
$$

Thus (3.6) yields

$$
\|A x-A w\|=\left\|A x-A w^{\prime}\right\|=\left\|A\left(x-w^{\prime}\right)\right\|=\left\|A u^{\prime \prime}\right\| \geq|A| \cdot\left\|u^{\prime \prime}\right\|=|A| \cdot\left\|x-w^{\prime}\right\| .
$$

This, when combined with (4.9) and the definition of the metric projection, yields

$$
d(A x, F)=\|A x-z\|=\|A x-A w\| \geq|A| \cdot\left\|x-w^{\prime}\right\| \geq|A| d(x, \text { Fix } \mathcal{L}\{T\}),
$$

which together with the equivalence (i) $\Leftrightarrow$ (iii) in Lemma 3.2 proves the second inequality in (4.7).

On the other hand, let $z=P_{\mathrm{Fix}} \mathcal{L}\{T\}$. Since $A z \in F$, we have

$$
d(x, \text { Fix } \mathcal{L}\{T\})=\|x-z\| \geq \frac{1}{\|A\|}\|A x-A z\| \geq \frac{1}{\|A\|} d(A x, F)
$$

and the proof is complete.

Corollary 4.5 Let $A: \mathcal{H}_{1} \rightarrow \mathcal{H}_{2}$ be a nonzero bounded linear operator with closed $\operatorname{im} A$ and let $Q \subseteq \mathcal{H}_{2}$ be closed and convex. Assume that $\operatorname{im} A \cap Q \neq \emptyset$. Then for any $x \in \mathcal{H}_{1}$, we have

$$
\frac{1}{\|A\|} d(A x, \operatorname{im} A \cap Q)=d\left(x, A^{-1}(Q)\right) \leq \frac{1}{|A|} d(A x, \operatorname{im} A \cap Q) .
$$

Proof. The result follows easily from Lemma 4.4 with $T=P_{Q}$.

Lemma 4.6 Let $A: \mathcal{H}_{1} \rightarrow \mathcal{H}_{2}$ be a nonzero bounded linear operator and let $T: \mathcal{H}_{2} \rightarrow \mathcal{H}_{2}$ be $\rho$-SQNE, where $\rho \geq 0$. Assume that $\operatorname{im} A \cap \operatorname{Fix} T \neq \emptyset$. Then for any $x \in \mathcal{H}_{1}$, we have

$$
\|T(A x)-A x\|^{2} \leq \frac{2\|A\|^{2}}{\rho+1}\|\mathcal{L}\{T\} x-x\| \cdot d(x, \operatorname{Fix} \mathcal{L}\{T\}) .
$$

Proof. Let $x \in \mathcal{H}_{1}$ and $z \in \operatorname{Fix} \mathcal{L}\{T\}$. We recall that, by Lemma 4.3, we have Fix $\mathcal{L}\{T\}=A^{-1}(\operatorname{Fix} T)$. Hence $A z \in \operatorname{Fix} T$. By Lemma 2.5 and by the Cauchy-Schwarz inequality, we have

$$
\begin{aligned}
\|T(A x)-A x\|^{2} & \leq \frac{2}{\rho+1}\langle T(A x)-A x, A z-A x\rangle \\
& =\frac{2\|A\|^{2}}{\rho+1}\left\langle\frac{1}{\|A\|^{2}} A^{*}(T(A x)-A x), z-x\right\rangle \\
& \leq \frac{2\|A\|^{2}}{\rho+1}\|\mathcal{L}\{T\} x-x\| \cdot\|z-x\| .
\end{aligned}
$$

Observe that for $z=P_{\mathrm{Fix} \mathcal{L}\{T\}} x$, we have $\|z-x\|=d(x$, Fix $\mathcal{L}\{T\})$, which completes the proof.

Theorem 4.7 Let $A: \mathcal{H}_{1} \rightarrow \mathcal{H}_{2}$ be a nonzero bounded linear operator, let $T: \mathcal{H}_{2} \rightarrow \mathcal{H}_{2}$ be $\rho$-SQNE, where $\rho \geq 0$, and let $S \subseteq \mathcal{H}_{2}$ be nonempty. Assume that $\operatorname{im} A \cap \operatorname{Fix} T \neq \emptyset$. Let $\mathcal{L}\{T\}: \mathcal{H}_{1} \rightarrow \mathcal{H}_{1}$ be the Landweber operator defined by (4.1). Then the following statements hold:

(i) If $T$ is weakly regular over $S$, then $\mathcal{L}\{T\}$ is weakly regular over $A^{-1}(S)$.

(ii) If $\operatorname{im} A$ is closed, $T$ is regular over $S, S$ is bounded and the family $\{\operatorname{im} A, \operatorname{Fix} T\}$ is regular over $S$, then $\mathcal{L}\{T\}$ is regular over $A^{-1}(S)$.

(iii) If $\operatorname{im} A$ is closed, $T$ is linearly regular with modulus $\delta>0$ over $S$ and the family $\{\operatorname{im} A, \operatorname{Fix} T\}$ is linearly regular with modulus $\kappa>0$ over $S$, then $\mathcal{L}\{T\}$ is linearly regular over $A^{-1}(S)$ with modulus

$$
\Delta:=\frac{\rho+1}{2}\left(\frac{\delta|A|}{\kappa\|A\|}\right)^{2}
$$

that is, for any $x \in A^{-1}(S)$, we have

$$
\|\mathcal{L}\{T\} x-x\| \geq \frac{\rho+1}{2}\left(\frac{\delta|A|}{\kappa\|A\|}\right)^{2} d(x, \operatorname{Fix} \mathcal{L}\{T\}) .
$$


Proof. For a bounded sequence $\left\{x_{k}\right\}_{k=0}^{\infty} \subseteq A^{-1}(S)$ and a point $z \in \operatorname{Fix} \mathcal{L}\{T\}$, we have

$$
\infty>R:=\sup _{k}\left\|x_{k}-z\right\| \geq d\left(x_{k}, \operatorname{Fix} \mathcal{L}\{T\}\right) .
$$

By (4.15) and (4.19), we obtain

$$
\begin{aligned}
\left\|T\left(A x_{k}\right)-A x_{k}\right\|^{2} & \leq \frac{2\|A\|^{2}}{\rho+1}\left\|\mathcal{L}\{T\} x_{k}-x_{k}\right\| \cdot d\left(x_{k}, \text { Fix } \mathcal{L}\{T\}\right) \\
& \leq \frac{2 R\|A\|^{2}}{\rho+1}\left\|\mathcal{L}\{T\} x_{k}-x_{k}\right\| .
\end{aligned}
$$

Part (i). Let $\left\{x_{k}\right\}_{k=0}^{\infty} \subseteq A^{-1}(S)$ and $x \in \mathcal{H}_{1}$ be such that

$$
x_{k} \rightarrow_{k} x \quad \text { and } \quad\left\|\mathcal{L}\{T\} x_{k}-x_{k}\right\| \rightarrow_{k} 0,
$$

and let $z \in \operatorname{Fix} \mathcal{L}\{T\}$. Since $\left\{x_{k}\right\}_{k=0}^{\infty}$ is bounded as a weakly convergent sequence, (4.20) and (4.21) yield $\left\|T\left(A x_{k}\right)-A x_{k}\right\| \rightarrow_{k} 0$. Observe that for any $y \in \mathcal{H}_{2}$, we get

$$
\left\langle A x_{k}-A x, y\right\rangle=\left\langle x_{k}-x, A^{*} y\right\rangle \rightarrow_{k} 0,
$$

and, consequently, $A x_{k} \rightarrow A x$. Since $A x_{k} \in S$, by the weak regularity of $T$, we obtain $A x \in$ Fix $T$. The latter statement is equivalent to $x \in A^{-1}(\operatorname{Fix} T)=\operatorname{Fix} \mathcal{L}\{T\}$, which completes the proof of part (i).

Part (ii). Let $S$ be bounded and let $\left\{x_{k}\right\}_{k=0}^{\infty} \subseteq A^{-1}(S)$ be such that

$$
\left\|\mathcal{L}\{T\} x_{k}-x_{k}\right\| \rightarrow_{k} 0 .
$$

By the second inequality in (4.7), the sequence $\left\{x_{k}\right\}_{k=0}^{\infty}$ is bounded. Using (4.20) and (4.23), we can conclude that

$$
\left\|T A x_{k}-A x_{k}\right\| \rightarrow_{k} 0
$$

Since $A x_{k} \in S$, using the regularity of the operator $T$ over $S$, we obtain

$$
\max \left\{d\left(A x_{k}, \operatorname{Fix} T\right), d\left(A x_{k}, \operatorname{im} A\right)\right\}=d\left(A x_{k}, \operatorname{Fix} T\right) \rightarrow_{k} 0 .
$$

By (4.7), (4.25) and by the regularity of the family $\{\operatorname{im} A, \operatorname{Fix} T\}$ over $S$, we arrive at

$$
d\left(x_{k}, \operatorname{Fix} \mathcal{L}\{T\}\right) \leq \frac{1}{|A|} d\left(A x_{k}, \operatorname{im} A \cap \operatorname{Fix} T\right) \rightarrow_{k} 0 .
$$

Note that $|A|>0$ is guaranteed by the assumption that $\operatorname{im} A$ is closed and by Lemma 3.2. It is clear that (4.26) completes the proof of part (ii).

Part (iii). Let $x \in A^{-1}(S)$ so that $A x \in S$. By the linear regularity of $T$ over $S$, the linear regularity of the family $\{\operatorname{im} A, \operatorname{Fix} T\}$ over $S$ and (4.7), we get

$$
\begin{aligned}
\|T(A x)-A x\| & \geq \delta d(A x, \operatorname{Fix} T) \\
& =\delta \max \{d(A x, \operatorname{Fix} T), d(A x, \operatorname{im} A)\} \\
& \geq \frac{\delta}{\kappa} d(A x, \operatorname{im} A \cap \operatorname{Fix} T) \\
& \geq \frac{\delta}{\kappa}|A| d(x, \operatorname{Fix} \mathcal{L}\{T\}) .
\end{aligned}
$$

Moreover, by (4.15),

$$
\|T(A x)-A x\|^{2} \leq \frac{2\|A\|^{2}}{\rho+1}\|\mathcal{L}\{T\} x-x\| \cdot d(x, \text { Fix } \mathcal{L}\{T\}),
$$

which, when combined with (4.27), leads to (4.18). This completes the proof. 
Remark 4.8 Assume that $T$ is a cutter $(\rho=1)$ and that all the assumptions of Theorem 4.7(iii) are satisfied. Let $\lambda \in(0,2]$. Then, for the relaxation $T_{\lambda}$ of $T$, inequality (4.18) takes the following form:

$$
\left\|\mathcal{L}\left\{T_{\lambda}\right\} x-x\right\|=\lambda\|\mathcal{L}\{T\} x-x\| \geq \lambda\left(\frac{\delta|A|}{\kappa\|A\|}\right)^{2} d\left(x, \operatorname{Fix} \mathcal{L}\left\{T_{\lambda}\right\}\right),
$$

where $x \in S$. In particular, if $T=P_{Q}$, where $Q \subseteq \mathcal{H}_{2}$ is nonempty, closed and convex, and $Q \cap \operatorname{im} A \neq \emptyset$, then

$$
\left\|\mathcal{L}_{\lambda}\left\{P_{Q}\right\} x-x\right\| \geq \lambda\left(\frac{|A|}{\kappa\|A\|}\right)^{2} d\left(x, A^{-1}(Q)\right) .
$$

Corollary 4.9 Let $A: \mathcal{H}_{1} \rightarrow \mathcal{H}_{2}$ be a nonzero bounded linear operator and let $T: \mathcal{H}_{2} \rightarrow \mathcal{H}_{2}$ be $\rho$-SQNE, where $\rho \geq 0$. Assume that $\operatorname{im} A \cap \operatorname{Fix} T \neq \emptyset$. Let $\mathcal{L}\{T\}: \mathcal{H}_{1} \rightarrow \mathcal{H}_{1}$ be the Landweber operator defined by (4.1). Then the following statements hold:

(i) If $T$ is weakly regular, then $\mathcal{L}\{T\}$ is weakly regular.

(ii) If $\operatorname{im} A$ is closed, $T$ is boundedly regular and $\{\operatorname{im} A, \operatorname{Fix} T\}$ is boundedly regular, then $\mathcal{L}\{T\}$ is boundedly regular.

(iii) If $\operatorname{im} A$ is closed, $T$ is boundedly linearly regular and the family $\{\operatorname{im} A, \operatorname{Fix} T\}$ is boundedly linearly regular, then $\mathcal{L}\{T\}$ is boundedly linearly regular.

Remark 4.10 Part (i) of Corollary 4.9 was proved in [Ceg15, Lemma 4.1]. This part can also be deduced from the proof of [WX11, Theorem 3.3]. Part (ii) of Corollary 4.9 was proved in [CM16, Theorem 4.2], under the assumption that $A$ is compact and $\underline{\lambda}:=\inf \Lambda^{+}\left(A^{*} A\right)>0$. Note that the latter result follows from Corollary 4.9(ii). Indeed, by the alternative (i) presented in Lemma 3.4, we see that $\underline{\lambda}>0$ only when $\operatorname{im} A$ is closed.

\section{Extrapolated Landweber Operator}

Let $T: \mathcal{H}_{2} \rightarrow \mathcal{H}_{2}$ be a given operator and $\sigma: \mathcal{H}_{1} \rightarrow[1, \infty)$ be an extrapolation function.

Definition 5.1 The operator $\mathcal{L}_{\sigma}\{T\}: \mathcal{H}_{1} \rightarrow \mathcal{H}_{1}$, defined by

$$
\mathcal{L}_{\sigma}\{T\} x:=x+\sigma(x)(\mathcal{L}\{T\} x-x), \quad x \in \mathcal{H}_{1},
$$

is called an extrapolated Landweber operator ( corresponding to $T$ and $\sigma$ ).

In this section, following [LMWX12] and [CM16] (see the Introduction), we consider the extrapolated Landweber operator $\mathcal{L}_{\sigma}\{T\}$ with $\sigma$ bounded from above by $\tau$ defined by

$$
\tau(x):= \begin{cases}\left(\frac{\|A\| \cdot\|T(A x)-A x\|}{\left\|A^{*}(T(A x)-A x)\right\|}\right)^{2}, & \text { if } A x \notin \operatorname{Fix} T, \\ 1, & \text { if } A x \in \operatorname{Fix} T .\end{cases}
$$

By (4.6), $\tau(x)$ and $\mathcal{L}_{\tau}\{T\} x$ are both well defined. Moreover, it is not difficult to see that $\tau(x) \geq 1$, since

$$
\left\|A^{*}(T(A x)-A x)\right\| \leq\|A\| \cdot\|T(A x)-A x\|
$$

and thus $\mathcal{L}_{\sigma}\{T\} x$ is also well defined.

Observe that when $\sigma=\tau$, then we have

$$
\mathcal{L}_{\tau}\{T\} x= \begin{cases}\left.x+\frac{\|T(A x)-A x\|^{2}}{\left\|A^{*}(T(A x)-A x)\right\|^{2}} A^{*}(T(A x)-A x)\right), & \text { if } A x \notin \operatorname{Fix} T, \\ x, & \text { if } A x \in \operatorname{Fix} T,\end{cases}
$$

that is, $\mathcal{L}_{\tau}\{T\} x$ does not depend on $\|A\|$. Similarly to Lemma 4.3, we have the following result. 
Lemma 5.2 Let $\mathcal{L}\{T\}$ be the Landweber operator corresponding to $T: \mathcal{H}_{2} \rightarrow \mathcal{H}_{2}, \lambda \in(0,1]$ and $\rho \geq 0$. Let $\sigma: \mathcal{H}_{1} \rightarrow[1, \infty)$ be an extrapolation function bounded from above by $\tau$ as defined in (5.2). If $T$ is $\rho$-SQNE and $\operatorname{im} A \cap \operatorname{Fix} T \neq \emptyset$, then $\mathcal{L}_{\lambda \sigma}\{T\}:=\operatorname{Id}+\lambda \sigma(\cdot)\left(\mathcal{L}_{\sigma}\{T\}-\mathrm{Id}\right)$ is also $\rho$-SQNE and we have $\operatorname{Fix} \mathcal{L}_{\lambda \sigma}\{T\}=A^{-1}(\operatorname{Fix} T)$.

Proof. The operator $\mathcal{L}_{\tau}\{T\}$ is $\rho$-SQNE by [CM16, Theorem 4.1]. Observe that by defining $\alpha(x):=$ $\lambda \frac{\sigma(x)}{\tau(x)} \in(0,1]$, we have $\mathcal{L}_{\lambda \sigma}\{T\} x=x+\alpha(x)\left(\mathcal{L}_{\tau}\{T\} x-x\right)$, that is, $\mathcal{L}_{\lambda \sigma}\{T\}$ is an $\alpha(\cdot)$-relaxation of $\mathcal{L}_{\tau}\{T\}$. Thus the result follows by (2.8) from Lemma 2.5.

Observe that for any point $x \in \mathcal{H}_{1}$, we have

$$
\left\|\mathcal{L}_{\sigma}\{T\} x-x\right\|=\sigma(x)\|\mathcal{L}\{T\} x-x\| \geq\|\mathcal{L}\{T\} x-x\| .
$$

This, when combined with either Theorem 4.7 or Corollary 4.9, leads to the following two results.

Theorem 5.3 Let $A: \mathcal{H}_{1} \rightarrow \mathcal{H}_{2}$ be a nonzero bounded linear operator, let $T: \mathcal{H}_{2} \rightarrow \mathcal{H}_{2}$ be $\rho$-SQNE, where $\rho \geq 0$ and let $S \subseteq \mathcal{H}_{2}$ be nonempty. Assume that $\operatorname{im} A \cap \operatorname{Fix} T \neq \emptyset$. Let $\mathcal{L}_{\sigma}\{T\}: \mathcal{H}_{1} \rightarrow \mathcal{H}_{1}$ be the extrapolated Landweber operator with $\sigma=\tau$, where $\tau$ is defined by (5.2). Then the following statements hold:

(i) If $T$ is weakly regular over $S$, then $\mathcal{L}_{\sigma}\{T\}$ is weakly regular over $A^{-1}(S)$.

(ii) If $\operatorname{im} A$ is closed, $T$ is regular over $S, S$ is bounded and $\{\operatorname{im} A, \operatorname{Fix} T\}$ is regular over $S$, then $\mathcal{L}_{\sigma}\{T\}$ is regular over $A^{-1}(S)$.

(iii) If $\operatorname{im} A$ is closed, $T$ is linearly regular with modulus $\delta>0$ over $S$ and the family $\{\operatorname{im} A$, Fix $T\}$ is linearly regular with modulus $\kappa>0$ over $S$, then $\mathcal{L}_{\sigma}\{T\}$ is linearly regular over $A^{-1}(S)$ with modulus $\Delta$ defined by (4.17). Moreover, for any $x \in A^{-1}(S)$, we have

$$
\left\|\mathcal{L}_{\sigma}\{T\} x-x\right\| \geq \sigma(x) \frac{\rho+1}{2}\left(\frac{\delta|A|}{\kappa\|A\|}\right)^{2} d\left(x, \operatorname{Fix} \mathcal{L}_{\sigma}\{T\}\right) .
$$

Corollary 5.4 Let $A: \mathcal{H}_{1} \rightarrow \mathcal{H}_{2}$ be a nonzero bounded linear operator and let $T: \mathcal{H}_{2} \rightarrow \mathcal{H}_{2}$ be $\rho$-SQNE, where $\rho \geq 0$. Assume that $\operatorname{im} A \cap \operatorname{Fix} T \neq \emptyset$. Let $\mathcal{L}_{\sigma}\{T\}: \mathcal{H}_{1} \rightarrow \mathcal{H}_{1}$ be the extrapolated Landweber operator with $\sigma=\tau$, where $\tau$ is defined by (5.2). Then the following statements hold:

(i) If $T$ is weakly regular, then $\mathcal{L}_{\sigma}\{T\}$ is weakly regular.

(ii) If $\operatorname{im} A$ is closed, $T$ is boundedly regular and the family $\{\operatorname{im} A, \operatorname{Fix} T\}$ is boundedly regular, then $\mathcal{L}_{\sigma}\{T\}$ is boundedly regular.

(iii) If $\operatorname{im} A$ is closed, $T$ is boundedly linearly regular and the family $\{\operatorname{im} A, \operatorname{Fix} T\}$ is boundedly linearly regular, then $\mathcal{L}_{\sigma}\{T\}$ is boundedly linearly regular.

Remark 5.5 As in Remark 4.8, assume that $T$ is a cutter $(\rho=1)$ and that all the assumptions of Theorem 5.3(iii) are satisfied. Let $\lambda \in(0,2]$. Then the relaxed Landweber operator $\mathcal{L}_{\lambda \sigma}\{T\}$ defined by

$$
\mathcal{L}_{\lambda \sigma}\{T\} x:=x+\lambda \sigma(x)(\mathcal{L}\{T\} x-x), \quad x \in \mathcal{H}_{1},
$$

is $\frac{2-\lambda}{\lambda}$-SQNE and satisfies

$$
\left\|\mathcal{L}_{\lambda \sigma}\{T\} x-x\right\|=\lambda \sigma(x)\|\mathcal{L}\{T\} x-x\| \geq \lambda \sigma(x)\left(\frac{\delta|A|}{\kappa\|A\|}\right)^{2} d\left(x, \text { Fix } \mathcal{L}_{\lambda \sigma}\{T\}\right),
$$

where $x \in S$. As in Remark 4.8, one can adjust the above inequality for $T=P_{Q}$, where $Q \subseteq \mathcal{H}_{2}$ is closed and convex, and $Q \cap \operatorname{im} A \neq \emptyset$. 


\section{Applications to the Split Convex Feasibility Problem}

In this section we propose a few $C Q$-type methods for solving the SCFP defined by (1.1)-(1.2), that is,

$$
\text { Find } x \in C=\text { Fix } S \text { such that } A x \in Q=\operatorname{Fix} T \text {, }
$$

where $S, T$ are given operators.

Theorem 6.1 Let $S: \mathcal{H}_{1} \rightarrow \mathcal{H}_{1}$ and $T: \mathcal{H}_{2} \rightarrow \mathcal{H}_{2}$ be $\rho_{S}$ - and $\rho_{T}-S Q N E$, respectively, where $\rho_{S}, \rho_{T}>0$. Let the sequence $\left\{x_{k}\right\}_{k=0}^{\infty}$ be defined by the method

$$
x_{0} \in \mathcal{H}_{1} ; \quad x_{k+1}:=S\left(x_{k}+\lambda_{k} \frac{\sigma\left(x_{k}\right)}{\|A\|^{2}} A^{*}\left(T\left(A x_{k}\right)-A x_{k}\right)\right),
$$

where the relaxation parameters satisfy $\lambda_{k} \in[\varepsilon, 1]$ for some $\varepsilon \in(0,1)$ and the extrapolation function $\sigma: \mathcal{H}_{1} \rightarrow[1, \infty)$ is bounded from above by $\tau$ as defined in $(5.2)$ (in particular, one can use $\sigma(x):=1$ or $\sigma(x):=\tau(x)$ for all $x)$. Assume that the solution set $F:=\operatorname{Fix} S \cap A^{-1}(\operatorname{Fix} T) \neq \emptyset$. Then the following statements hold:

(i) If $S$ and $T$ are both weakly regular, then the sequence $\left\{x_{k}\right\}_{k=0}^{\infty}$ weakly converges to some $x_{\infty} \in F$.

(ii) If $\operatorname{im} A$ is closed, $S$ and $T$ are both boundedly regular, and the two families of sets $\{\operatorname{im} A$, Fix $T\}$ and $\left\{\right.$ Fix $\left.S, A^{-1}(\operatorname{Fix} T)\right\}$ are boundedly regular, then the convergence to $x_{\infty}$ is in norm.

(iii) If im $A$ is closed, $S$ and $T$ are both boundedly linearly regular, and the following two families of sets $\{\operatorname{im} A, \operatorname{Fix} T\}$ and $\left\{\operatorname{Fix} S, A^{-1}(\operatorname{Fix} T)\right\}$ are boundedly linearly regular, then the convergence to $x_{\infty}$ is at least linear, that is,

$$
d\left(x_{k+1}, F\right) \leq q d\left(x_{k}, F\right) \quad \text { and } \quad\left\|x_{k}-x_{\infty}\right\| \leq 2 d\left(x_{0}, F\right) q^{k}
$$

for some $q \in(0,1)$, which may depend on $x_{0}$.

Proof. Observe that since we can write $x_{k+1}=S \mathcal{L}_{\lambda_{k} \sigma}\{T\} x_{k}$, the sequence $\left\{x_{k}\right\}_{k=0}^{\infty}$ is Fejér monotone with respect to $F$. Indeed, by Lemma 5.2, the operator $\mathcal{L}_{\lambda \sigma}\{T\}$ is $\rho_{T}$-SQNE and Fix $\mathcal{L}_{\lambda \sigma}\{T\}=$ $A^{-1}($ Fix $T)$. By Theorem 2.6, the product $\left.S \mathcal{L}_{\lambda_{k}} \sigma T\right\}$ is $\left(\frac{1}{2} \min \left\{\rho_{S}, \rho_{T}\right\}\right)$-SQNE with Fix $\left.S \mathcal{L}_{\lambda_{k}} \sigma T\right\}=F$. Hence for any point $z \in F$, we have

$$
\left\|x_{k+1}-z\right\|^{2} \leq\left\|x_{k}-z\right\|^{2}-\frac{\min \left\{\rho_{S}, \rho_{T}\right\}}{2}\left\|x_{k+1}-x_{k}\right\|^{2} .
$$

Moreover, since $\left\{\left\|x_{k}-z\right\|\right\}_{k=0}^{\infty}$ converges as a decreasing sequence, we have

$$
\left\|S \mathcal{L}_{\lambda_{k} \sigma} x_{k}-x_{k}\right\|=\left\|x_{k+1}-x_{k}\right\| \rightarrow 0 .
$$

By Fejér monotonicity, we also have

$$
\left\{x_{k}\right\} \subset B_{1}:=\left\{x \in \mathcal{H}_{1} \mid\left\|x-P_{F} x_{0}\right\| \leq r:=d\left(x_{0}, F\right)\right\}
$$

Consequently,

$$
\left\{A x_{k}\right\} \subset B_{2}:=\left\{y \in \mathcal{H}_{2} \mid\left\|y-A P_{F} x_{0}\right\| \leq r\|A\|\right\}
$$

and

$$
B_{1} \subseteq A^{-1}\left(B_{2}\right) .
$$

The remaining part of the proof follows from Theorems 2.2, 2.12 and 5.3.

Part (i). By assumption, $S$ is weakly regular over $B_{1}$ and $T$ is weakly regular over $B_{2}$. By Theorem 5.3 (i) and by (6.8), the operator $\mathcal{L}_{\sigma}\{T\}$ is weakly regular over $B_{1}$. It is not difficult to see that the sequence of relaxations $\left\{\mathcal{L}_{\lambda_{k} \sigma}\right\}_{k=0}^{\infty}$ is also weakly regular over $B_{1}$ (see [CRZ18, Proposition 4.7]). Hence, by Theorem $2.12(\mathrm{i})$, the product sequence $\left\{S \mathcal{L}_{\lambda_{k} \sigma}\right\}_{k=0}^{\infty}$ is weakly regular over $B_{1}$ as well. Thus (by 
the definition of weak regularity) any weak cluster point of $\left\{x_{k}\right\}_{k=0}^{\infty}$ is in $F$, which shows that $\left\{x_{k}\right\}_{k=0}^{\infty}$ converges weakly to some point $x_{\infty} \in F$ in view of Theorem 2.2 (i).

Part (ii). Using an argument similar to the one above, we conclude that $\left\{\mathcal{L}_{\lambda_{k} \sigma}\right\}_{k=0}^{\infty}$ is regular over $B_{1}$. By applying Theorem 2.12 (ii), we see that $\left\{S \mathcal{L}_{\lambda_{k} \sigma}\right\}_{k=0}^{\infty}$ is also regular over $B_{1}$. Hence by (6.5), when combined with the definition of regularity over $B_{1}$, we see that $d\left(x_{k}, F\right) \rightarrow 0$, which implies that $\left\|x_{k}-x_{\infty}\right\| \rightarrow 0$ in view of Theorem 2.2 (ii).

Part (iii). By assumption, the operator $T$ and the family $\{\operatorname{im} A, \operatorname{Fix} T\}$ are both linearly regular over $B_{2}$ with moduli $\delta_{T}$ and $\kappa_{2}$, respectively. Thus, by Theorem 5.3 (iii) and (6.8), the operator $\mathcal{L}_{\sigma}\{T\}$ is linearly regular over $B_{1}$ with modulus $\Delta$ defined in (4.17) (with $\kappa:=\kappa_{2}$ ). Consequently, the sequence $\left\{\mathcal{L}_{\lambda_{k} \sigma}\{T\}\right\}_{k=0}^{\infty}$ is linearly regular over the same ball with modulus $\varepsilon \Delta$.

By another assumption, the family $\left\{\operatorname{Fix} S, A^{-1}(\operatorname{Fix} T)\right\}$ is linearly regular over $B_{1}$ with modulus $\kappa_{1}$. By applying Theorem 2.12 (iii) to $\{S\}_{k=0}^{\infty}$ and $\left\{\mathcal{L}_{\lambda_{k} \sigma}\{T\}\right\}_{k=0}^{\infty}$, we conclude that the product sequence $\left\{S \mathcal{L}_{\lambda_{k} \sigma}\right\}_{k=0}^{\infty}$ is also linearly regular over $B_{1}$ with modulus

$$
\Gamma=\min \left\{\rho_{S}, \rho_{T}\right\}\left(\frac{\min \left\{\delta_{S}, \varepsilon \Delta\right\}}{2 \kappa_{1}}\right)^{2}
$$

that is, $\left\|x_{k+1}-x_{k}\right\| \geq \Gamma d\left(x_{k}, F\right)$. Hence, by setting $z:=P_{F} x_{k}$ in (6.4) and by the inequality $d\left(x_{k+1}, F\right) \leq$ $\left\|x_{k+1}-P_{F} x_{k}\right\|$, we arrive at

$$
d^{2}\left(x_{k+1}, F\right) \leq d^{2}\left(x_{k}, F\right)-\frac{\min \left\{\rho_{S}, \rho_{T}\right\}}{2} \Gamma^{2} d^{2}\left(x_{k}, F\right) .
$$

Consequently, $d\left(x_{k+1}\right) \leq q d\left(x_{k}, F\right)$ with

$$
q:=\sqrt{1-\frac{\min \left\{\rho_{S}, \rho_{T}\right\}}{2} \Gamma^{2}}
$$

and, by Theorem 2.2(iii), we also have $\left\|x_{k}-x_{\infty}\right\| \leq 2 d\left(x_{0}, F\right) q^{k}$. This completes the proof.

Note again, as in (5.4), that applying $\sigma(x)=\tau(x)$ in (6.2) with $\tau(x)$ defined by (5.2), we do not need to know the norm of $A$.

Remark 6.2 (Cutters $S$ and $T$ ) If we assume that both $S$ and $T$ are cutters, then the relaxation parameters $\lambda_{k}$ can be chosen from the interval $[\varepsilon, 2-\varepsilon]$ for some $\varepsilon \in(0,1)$. Indeed, define $U:=$ $\mathrm{Id}+(2-\varepsilon)(T-\mathrm{Id})$ and $\alpha_{k}:=\frac{\lambda_{k}}{2-\varepsilon}$. Then $U$ is $\frac{\varepsilon}{2-\varepsilon}$-SQNE, $\alpha_{k} \in\left[\frac{\varepsilon}{2-\varepsilon}, 1\right]$ and $S \mathcal{L}_{\lambda_{k} \sigma}\{T\} x=S \mathcal{L}_{\alpha_{k} \sigma}\{U\} x$. Moreover, the upper bound for $\sigma$ defined by (5.2) is exactly the same as the corresponding upper bound determined by $U$, that is, for $A x \notin \operatorname{Fix} T=\operatorname{Fix} U$, we have

$$
\tau(x)=\left(\frac{\|A\| \cdot\|T(A x)-A x\|}{\left\|A^{*}(T(A x)-A x)\right\|}\right)^{2}=\left(\frac{\|A\| \cdot\|U(A x)-A x\|}{\left\|A^{*}(U(A x)-A x)\right\|}\right)^{2} .
$$

Consequently, the sequence defined by

$$
x_{0} \in \mathcal{H}_{1} ; \quad x_{k+1}:=S \mathcal{L}_{\lambda_{k} \sigma}\{T\} x_{k}
$$

is a particular case of the iteration defined in Theorem 6.1. In addition, the convergence statements (i), (ii) and (iii) hold here as well since weak/bounded/bounded linear regularity of $T$ implies the same type of regularity for $U$.

Corollary 6.3 (Extrapolated $C Q$-method) Let $C \subseteq \mathcal{H}_{1}$ and $Q \subseteq \mathcal{H}_{2}$ be nonempty, closed and convex. Let the sequence $\left\{x_{k}\right\}_{k=0}^{\infty}$ be defined by the method

$$
x_{0} \in \mathcal{H}_{1} ; \quad x_{k+1}:=P_{C}\left(x_{k}+\lambda_{k} \frac{\sigma\left(x_{k}\right)}{\|A\|^{2}} A^{*}\left(P_{Q}\left(A x_{k}\right)-A x_{k}\right)\right),
$$


where the relaxation parameters satisfy $\lambda_{k} \in[\varepsilon, 2-\varepsilon]$ for some $\varepsilon \in(0,1)$ and the extrapolation function $\sigma: \mathcal{H}_{1} \rightarrow[1, \infty)$ is bounded from above by $\tau$ defined by (compare with (5.2))

$$
\tau(x):= \begin{cases}\left(\frac{\|A\| \cdot\left\|P_{Q}(A x)-A x\right\|}{\left\|A^{*}\left(P_{Q}(A x)-A x\right)\right\|}\right)^{2}, & \text { if } A x \notin Q, \\ 1, & \text { if } A x \in Q ;\end{cases}
$$

(in particular, one can use $\sigma(x):=1$ or $\sigma(x):=\tau(x)$ for all $x$ ). Assume that $F:=C \cap A^{-1}(Q) \neq \emptyset$. Then the following statements hold:

(i) The sequence $\left\{x_{k}\right\}_{k=0}^{\infty}$ weakly converges to some $x_{\infty} \in F$.

(ii) If $\operatorname{im} A$ is closed, and the two families of sets $\{\operatorname{im} A, Q\}$ and $\left\{C, A^{-1}(Q)\right\}$ are boundedly regular, then the convergence to $x_{\infty}$ is in norm.

(iii) If $\operatorname{im} A$ is closed, and the two families of sets $\{\operatorname{im} A, Q\}$ and $\left\{C, A^{-1}(Q)\right\}$ are boundedly linearly regular, then the convergence to $x_{\infty}$ is at least linear, that is,

$$
d\left(x_{k+1}, F\right) \leq q d\left(x_{k}, F\right) \quad \text { and } \quad\left\|x_{k}-x_{\infty}\right\| \leq 2 d\left(x_{0}, F\right) q^{k},
$$

for some $q \in(0,1)$, which may depend on $x_{0}$.

Proof. The projections $P_{C}$ and $P_{Q}$ are linearly regular cutters. Recall that linear regularity implies regularity and this in turn implies weak regularity; see [CRZ18, Corollary 4.4]. Hence, the result follows from Theorem 6.1 and Remark 6.2.

Example 6.4 Assume that $\operatorname{im} A$ is closed. Then the two additional regularity conditions mentioned in Corollary 6.3 (iii) are satisfied if, for example, $C \cap \operatorname{int} A^{-1}(Q) \neq \emptyset$, or when $A(C) \cap \operatorname{int} Q \neq \emptyset$. This follows from Example 2.8 (iii).

Corollary 6.5 (Extrapolated-subgradient $C Q$ method) Let $C=\left\{x \in \mathcal{H}_{1} \mid c(x) \leq 0\right\} \neq \emptyset$ and $Q=\left\{y \in \mathcal{H}_{2} \mid q(x) \leq 0\right\} \neq \emptyset$ for some lower semi-continuous and convex functions $c$ and $q$. Let the sequence $\left\{x_{k}\right\}_{k=0}^{\infty}$ be defined by the method

$$
x_{0} \in \mathcal{H}_{1} ; \quad x_{k+1}:=P_{c}\left(x_{k}+\lambda_{k} \frac{\sigma\left(x_{k}\right)}{\|A\|^{2}} A^{*}\left(P_{q}\left(A x_{k}\right)-A x_{k}\right)\right),
$$

where $P_{c}$ and $P_{q}$ are subgradient projections (see Example 2.4), the relaxation parameters satisfy $\lambda_{k} \in$ $[\varepsilon, 2-\varepsilon]$ for some $\varepsilon \in(0,1)$ and the extrapolation function $\sigma: \mathcal{H}_{1} \rightarrow[1, \infty)$ is bounded from above by $\tau$ defined by (compare with (5.2))

$$
\tau(x):= \begin{cases}\left(\frac{\|A\| \cdot\left\|P_{q}(A x)-A x\right\|}{\left\|A^{*}\left(P_{q}(A x)-A x\right)\right\|}\right)^{2}, & \text { if } q(A x)>0, \\ 1, & \text { if } q(A x) \leq 0 ;\end{cases}
$$

(in particular, one can use $\sigma(x):=1$ or $\sigma(x):=\tau(x)$ for all $x$ ). Assume that $F:=C \cap A^{-1}(Q) \neq \emptyset$, and that $c$ and $q$ are Lipschitz continuous on bounded sets (see Example 2.10 and Remark 2.11). Then the following statements hold:

(i) The sequence $\left\{x_{k}\right\}_{k=0}^{\infty}$ weakly converges to some $x_{\infty} \in F$.

(ii) If $\mathrm{im} A$ is closed, $P_{c}$ and $P_{q}$ are both boundedly regular (for example, when both functions $c$ and $q$ are strongly convex) and the two families of sets $\{\mathrm{im} A, Q\}$ and $\left\{C, A^{-1}(Q)\right\}$ are boundedly regular, then the convergence to $x_{\infty}$ is in norm.

(iii) If $\mathrm{im} A$ is closed, $P_{c}$ and $P_{q}$ are both boundedly linearly regular and the two families of sets $\{\mathrm{im} A, Q\}$ and $\left\{C, A^{-1}(Q)\right\}$ are boundedly linearly regular, then the convergence to $x_{\infty}$ is at least linear, that is,

$$
d\left(x_{k+1}, F\right) \leq q d\left(x_{k}, F\right) \quad \text { and } \quad\left\|x_{k}-x_{\infty}\right\| \leq 2 d\left(x_{0}, F\right) q^{k}
$$

for some $q \in(0,1)$ which may depend on $x_{0}$. 
Example 6.6 All the regularity conditions mentioned in Corollary 6.5 (iii) are satisfied if, for example, there is $z \in \mathcal{H}_{1}$ such that $c(z)<0$ and $q(A z)<0$. This follows from Examples 2.8(iii) and 2.10(iii).

Remark 6.7 (Bounded linear regularity of the SCFP) Conditions presented in Theorem 6.1 (iii) and in Corollary 6.3 (iii) imply that the split convex feasibility problem has the bounded linear regularity property in the sense of [WHLY17, Definition 2.2]; compare with (1.15). Indeed, by assumption, $\left\{C, A^{-1}(Q)\right\}$ is $\kappa_{1}$-linearly regular over $B_{1}:=\left\{x \in \mathcal{H}_{1} \mid\|x\| \leq r\right\}$ and $\{\operatorname{im} A, Q\}$ is $\kappa_{2}$-linearly regular over $B_{2}:=\left\{y \in \mathcal{H}_{2} \mid\|y\| \leq\|A\| r\right\}$. Consequently, for any $x \in C \cap B_{1}$, we have $A x \in B_{2}$ and thus, by Lemma 4.4,

$$
d(A x, Q) \geq \frac{1}{\kappa_{2}} d(A x, \operatorname{im} A \cap Q) \geq \frac{|A|}{\kappa_{2}} d\left(x, A^{-1}(Q)\right) \geq \frac{|A|}{\kappa_{1} \kappa_{2}} d\left(x, C \cap A^{-1}(Q)\right) .
$$

At this point, it is worth emphasizing that Theorem 6.1 also applies to operators other than projections.

Acknowledgement. This research was supported in part by the Israel Science Foundation (Grants no. 389/12 and 820/17), by the Fund for the Promotion of Research at the Technion and by the Technion General Research Fund.

\section{References}

[BBR78] J. B. Baillon, R. E. Bruck, S. Reich, On the asymptotic behabviour of the nonexpansive mappings in banach spaces, Houston J. Math., 4 (1978), 1-9.

[BKRZ18] C. Bargetz, V. I. Kolobov, S. Reich, R. Zalas, Linear convergence rates for extrapolated fixed point algorithms, Optimization, DOI:10.1080/02331934.2018.1512109.

[BB96] H. H. Bauschke and J. Borwein, On projection algorithms for solving convex feasibility problems, SIAM Review, 38 (1996), 367-426.

[BNP15] H. H. Bauschke, D. Noll, H. M. Phan, Linear and strong convergence of algorithms involving averaged nonexpansive operators, J. Math. Anal. Appl., 421 (2015) 1-20.

[BC17] H. H. Bauschke, P. L. Combettes, Convex Analysis and Monotone Operator Theory in Hilbert Spaces, Second edition, Springer, Cham, 2017.

[Byr02] C. Byrne, Iterative oblique projection onto convex sets and the split feasibility problem, Inverse Problems, 18 (2002), pp. 441-453.

[Byr04] C. Byrne, A unified treatment of some iterative algorithms in signal processing and image reconstruction, Inverse Problems, 20 (2004), 103-120.

[Ceg12] A. Cegielski, Iterative Methods for Fixed Point Problems in Hilbert Spaces, Lecture Notes in Mathematics, 2057, Springer, Heidelberg, 2012.

[Ceg15] A. Cegielski, General method for solving the split common fixed point problem, J. Optim. Theory Appl., 165 (2015), 385-404.

[Ceg16] A. Cegielski, Landweber-type operator and its properties, Contemporary Mathematics, 658 (2016), 139-148.

[CM16] A. Cegielski, F. Al-Musallam, Strong convergence of a hybrid steepest descent method for the split common fixed point problem, Optimization, 65 (2016), 1463-1476.

[CRZ18] A. Cegielski, S. Reich, R. Zalas, Regular sequences of quasi-nonexpansive operators and their applications, SIAM Journal on Optimization, 28 (2018), 1508-1532.

[CE94] Y. Censor, T. Elfving, A multiprojection algorithm using Bregman projections in a product space, Numer. Algorithms, 8 (1994), 221-239. 
[CEKB05] Y. Censor, T. Elfving, N. Kopf, T. Bortfeld, The multiple-sets split feasibility problem and its applications for inverse problems, Inverse Problems, 21 (2005), 2071-2084.

[CMS07] Y. Censor, A. Motova, A. Segal, Perturbed projections and subgradient projections for the multiple-sets split feasibility problem, J. Math. Anal. Appl., 327 (2001), 1244-1256.

[CS09] Y. Censor, A. Segal, The split common fixed point problem for directed operators, J. Convex Anal., 16 (2009), 587-600.

[DM05] L. Debnath and P. Mikusiński, Hilbert Spaces with Applications, Third Edition, Elsevier, Amsterdam, 2005.

[Deu01] F. Deutsch, Best Approximation in Inner Product Spaces, Springer-Verlag, New York, 2001.

[JEKC06] B. Johansson, T. Elfving, V. Kozlov, Y. Censor, P.-E. Forssén, G. Granlund, The application of an oblique-projected Landweber method to a model of supervised learning, Mathematical and Computer Modelling, 43 (2006), 892-909.

[Kra55] M. A. Krasnosel'skiı̌, Two remarks on the method of successive approximations, Uspekhi Mat. Nauk 10, (1955), 123-127.

[Kre14] R. Kress, Linear Integral Equations, Third edition, Applied Mathematical Sciences, 82, Springer, New York, 2014.

[Lan51] L. Landweber, An iterative formula for Fredholm integral equations of the first kind, Am. J. Math., 73 (1951), 615-24.

[LMWX12] G. López, V. Martín-Márquez, F. Wang, H.-K. Xu, Solving the split feasibility problem without prior knowledge of matrix norms, Inverse Problems, 28 (2012), 085004 (18pp).

[Man53] W. R. Mann, Mean value methods in iteration, Proc. Am. Math. Soc., 4 (1953), 506-510.

[MR07] E. Masad, S. Reich, A note on the multiple-set split convex feasibility problem in Hilbert space, J. Nonlinear Convex Anal., 8 (2007), 367-371.

[Mou11] A. Moudafi, A note on the split common fixed-point problem for quasi-nonexpansive operators, Nonlinear Anal., 74 (2011), 4083-4087.

[PB97] M. Piana, M. Bertero, Projected Landweber method and preconditioning, Inverse Problems, 13 (1997), 441-463.

[Pol87] B. T. Polyak, Introduction to Optimization, Optimization Software, New York, 1987.

[QX05] B. Qu, N. Xiu, A note on the CQ algorithm for the split feasibility problem, Inverse Problems, 21 (2005), 1655-1665.

[WX11] F. Wang, H.-K. Xu, Cyclic algorithms for split feasibility problems in Hilbert spaces. Nonlinear Analysis, 74 (2011), 4105-4111.

[WHLY17] J. Wang, Y. Hu, C. Li, J.-C. Yao, Linear convergence of CQ algorithms and applications in gene regulatory network inference, Inverse Problems, 33 (2017), 055017 (25pp).

[Xu06] H.-K. Xu, A variable Krasnosel'skiı-Mann algorithm and the multiple-set split feasibility problem, Inverse Problems, 22 (2006), 2021-2034.

[Xu10] H.-K. Xu, Iterative methods for the split feasibility problem in infinite-dimensional Hilbert spaces, Inverse Problems, 26 (2010), 105018 (17pp).

[Xu11] H.-K. Xu, Averaged mappings and the gradient-projection algorithm, J. Optim. Theory Appl., 50 (2011), 360-378. 
[Yan04] Q. Yang, The relaxed CQ algorithm solving the split feasibility problem, Inverse Problems, 20 (2004), 1261-1266.

[Yan05] Q. Yang, On variable-step relaxed projection algorithm for variational inequalities, J. Math. Anal. Appl., 302 (2005), 166-179.

[Yos78] K. Yosida, Functional Analysis, Fifth Edition, Springer-Verlag, Berlin, 1978.

[ZC10] H. Zhang and L. Z. Cheng, Projected Landweber iteration for matrix completion, Journal of Computational and Applied Mathematics, 235 (2010), 593-601. 